\title{
The ergoregion in the Kerr spacetime: properties of the equatorial circular motion
}

\author{
D. Pugliese ${ }^{1,2, a}$, H. Quevedo Q $^{3,4, b}$ \\ ${ }^{1}$ Faculty of Philosophy and Science, Institute of Physics, Silesian University in Opava, Bezručovo náměstí 13, 74601 Opava, Czech Republic \\ ${ }^{2}$ School of Mathematical Sciences, Queen Mary University of London, Mile End Road, London E1 4NS, UK \\ ${ }^{3}$ Instituto de Ciencias Nucleares, Universidad Nacional Autónoma de México, AP 70543, 04510 México, DF, Mexico \\ ${ }^{4}$ Dipartimento di Fisica, Università di Roma "La Sapienza", Piazzale Aldo Moro 5, 00185 Roma, Italy
}

Received: 24 March 2015 / Accepted: 12 May 2015 / Published online: 29 May 2015

(C) The Author(s) 2015. This article is published with open access at Springerlink.com

\begin{abstract}
We investigate in detail the circular motion of test particles in the equatorial plane of the ergoregion in the Kerr spacetime. We consider all the regions where circular motion is allowed, and we analyze the stability properties and the energy and angular momentum of the test particles. We show that the structure of the stability regions has definite features that make it possible to distinguish between black holes and naked singularities. The naked singularity case presents a very structured non-connected set of regions of orbital stability, where the presence of counterrotating particles and zero angular momentum particles for a specific class of naked singularities is interpreted as due to the presence of a repulsive field generated by the central source of gravity. In particular, we analyze the effects of the dynamical structure of the ergoregion (the union of the orbital regions for different attractor spins) on the behavior of accretion disks around the central source. The properties of the circular motion turn out to be so distinctive that they allow for the introduction of a complete classification of Kerr spacetimes, each class of which is characterized by different physical effects that could be of especial relevance in observational astrophysics. We also identify some special black-hole spacetimes where these effects could be relevant.
\end{abstract}

\section{Introduction}

Black holes are very probably the central engines of quasars, active galactic nuclei, and gamma ray bursts. Consequently, the mechanism by which energy is extracted from them is of great astrophysical interest. While the exact form of this mechanism is not known, it seems that the effects occurring

\footnotetext{
a e-mail: d.pugliese.physics@gmail.com

b e-mail: quevedo@nucleares.unam.mx
}

inside the ergoregion of black holes are essential for understanding the central engine mechanism $[1,2]$.

On the other hand, since the discussion first presented in [3], the issue of the creation and the stability of naked singularities has been intensively debated with different controversial results [4-7]. For instance, Ref. [8] addresses the issue of the possible formation of a naked singularity, analyzing the stability of the progenitor models and investigating the gravitational collapse of differentially rotating neutron stars (in full general relativity). These results do not exclude the possibility that a naked singularity can be produced as the result of a gravitational collapse, under quite general conditions on the progenitor and considering instability processes. Since practically all these results are based upon a numerical integration of the corresponding field equations, their interpretation requires a careful analysis. Indeed, the formation of black holes (with singularities inside the horizon) is usually associated with the existence of trapped surfaces. So, the numerical detection of a singularity without trapped surfaces is usually considered as a proof that the singularity is naked. This, however, is not always true. As has been shown in [63], during the formation of a spherically symmetric black hole it is possible to choose a very particular slicing of spacetime such that no trapped surfaces exist. This shows that the nonexistence of trapped surfaces in the gravitational collapse cannot be considered in general as a proof of the existence of a naked singularity. A more detailed analysis (further numerical integration) is necessary in order to establish that a naked singularity can be formed as the end result of a gravitational collapse. See, for instance, [64] for particular examples. In any case, it is therefore interesting to investigate the physical effects associated with the gravitational field of naked singularities.

In astrophysics, it is particularly interesting to study the general features of the motion of test particles moving along 
circular orbits around the central source. In fact, one can imagine a thin disk made only of test particles as a hypothetical accretion disk of matter surrounding the central source. Although this is a very idealized model for an accretion disk, one can nevertheless extract some valuable information as regards the dynamics of particles in the corresponding gravitational field and the amount of energy that can be released by matter when falling into the central mass distribution (see [9] and [10,14-18]), with the equatorial circular geodesics being then relevant for the Keplerian accretion disks. Moreover, there is an increasing interest in the properties of the matter dynamics in the ergoregion, especially as a possible source of data discriminating between black holes and superspinning objects (see [19-24]). In [19,25-28], we studied the geometric structure of such an idealized accretion disk and how it can depend on the values of the physical parameters that determine the gravitational field. This is also an important issue, since it could lead to physical effects that depend on the structure of the accretion disk, with the corresponding possible observational consequences. Indeed, in a series of previous studies $[19,25-28]$, it was established that the motion of test particles in the equatorial plane of black-hole spacetimes can be used to derive information as regards the structure of the central source of gravitation (see also discussions in [21,29,30] and [31]). In [19] and [28], some of the characteristics of the circular motion in the Kerr and KerrNewman spacetimes for black holes and naked singularities were discussed, and the typical effects of repulsive gravity were revealed in the naked singularity ergoregion (see also [20-24]). Moreover, it was pointed out that there exists a dramatic difference in black holes and naked singularities with respect to the zero and negative energy states in circular orbits. In this work, we clarify and deepen those results, providing a classification of attractor sources on the basis of the properties of this type of dynamics; we focus here exclusively on the dynamics within the ergoregion, considering bounded and unbounded unstable orbits, and investigate the main possible astrophysical implications with particular attention to the physics of accretion disks. Our aim is to explore the dynamics within this region from the point of view of the orbiting matter to enlighten also the instabilities that could give rise to detectable phenomena in astrophysics. This analysis has two essential features. First, from the methodological standpoint we introduce the notion of dynamical structure of the ergoregion, which is essentially a spacetime region decomposition based on the geometric properties investigated here, and, secondly, we connect in a quite natural manner our study to the analysis of configurations of extended toroidal matter in accretion such as accretion disk thick models, where the equilibrium and the unstable states of the system are mainly governed by the curvature effects of the geometry. The relevance of these studies in relation to the (hydrodynamic) relativistic disk models had been anticipated in [34], where, however, the regions near the static limit remained unexplored. Here, we revisit these considerations, analyze the static limit, and focus our attention on equilibrium configurations as well as on the location and dynamics of unstable configurations with the associated accretion or launch of jets.

From the methodological point of view our approach consists in analyzing the behavior of the energy and angular momentum of the test particles as functions of the radial distance and of the intrinsic angular momentum of the central source, which for the sake of brevity will be often referred as the "spin" of the source. This procedure allows us to carry out a methodical and detailed physical analysis of all the regions inside the ergoregion where circular motion is allowed. We investigate the dynamics inside the ergoregion, defining and characterizing the regions where there are stable orbits, bounded or unbounded unstable orbits, and, eventually, the regions where particles with negative energies are allowed. The disjoint union of these orbital sections fully covers the ergoregion and the set of regions defines the dynamical structure or decomposition of the ergoregion, fully characterizing indeed the dynamical properties of the orbiting matter. We study the dynamical structure of the ergoregion and the behavior of matter in accretion, identifying different scenarios where there may be potentially detectable effects. We discuss the possible astrophysical implications of this analysis in terms of the dynamical structure, particularly in relation with the source evolution and the configuration of the extended matter. The dynamical structure plays in fact a specific and fundamental role in the modeling of accretion disks. The properties of matter in circular configurations, i.e., toroidal configurations of extended matter in accretion, are in general governed by several factors such as the pressure, the magnetic fields, the dissipative effects, etc., but the properties of the spacetime structure highlighted by the geodesics motion are the basis of any disk model. In particular, we refer here to the relativistic models of pressure supported disks where the roles of the hydrostatic pressure and the background are crucial for determining both the equilibrium phases and the gravitational and hydrostatic instabilities, leading to the accretion or the launch of jets in funnels of matter from the instability regions [32-34].

In general, one might look at the entire evolution of a thick accretion disk, from the formation of a thin ring, its growing to the unstable phases of the accretion, resulting finally in the formation of jets of matter from the cusp point, as the orbiting matter transition in the dynamical structure of the ergoregion. As the disk equatorial plane of symmetry often coincides with the symmetric plane of the accretor, by using the symmetry of the system we can reduce the analysis to a one-dimensional problem along the radial direction, the dynamics of the orbiting matter then being regulated entirely by a couple of model parameters, so that the main features of 
the disk dynamics, stability, and even the accretion are mainly regulated by the configuration properties on the plane of symmetry. We will see that from this analysis different classes of attractors emerge. Moreover, we will show that there are certain similarities in the dynamical structure of the ergoregions of naked singularities with sufficiently high spins, strong naked singularities, and black holes with sufficiently small spin, i.e., weak black holes, in the sense that none of them allow for any stable orbiting matter inside the ergoregion. In addition, our analysis shows that an increase of the attractor spin in weak black-hole geometries, or a decrease in strong and very strong naked singularities, affects positively the process of the formation of a toroidal orbiting configuration. We also perform a detailed classification of these sources, taking into account the different dynamical structures and the peculiarities of the limiting geometries defined as the boundaries of the different classes. We ultimately conjecture that these peculiar spacetimes could be involved in spin-up and -down processes that lead to a radical change of the dynamical structure of the region closest to the source and, therefore, potentially could give rise to detectable effects.

However, it has been argued, for example in [35], that the ergoregion cannot disappear as a consequence of a spin shift, as it could be filled by negative energy matter produced by a Penrose process [36]. In the case of a naked singularity, this process is combined with the repulsive effects of the singularity ring. Since a set of particles with negative energy could exist in the ergoregion, one can argue about their fate and role in the evolution of the source. These very peculiar particles differ fundamentally as regards the two types of singularities from the point of view of the circular motion and their stability: the very weak naked singularities are distinguished by the existence of counterrotating orbits. The existence of these very orbits can be seen as a "repulsive gravity" effect that has been shown to exist also in other spacetimes with naked singularities [25,26,28]. This is a characteristic feature of the naked singularity geometries [37-39]. In this work, we identify the repulsion effect and its role in the determination of the dynamical structure of the ergoregion and the extended matter in accretion. This region creates therefore an "antigravity" sphere bounded by orbits with zero angular momentum. In a bounded region in the antigravity sphere, setting a bubble of trapped (bounded or stable) negative energy particles may form stable or unstable toroidal configurations, explaining, at least for very weak naked singularities, the fate of particles with negative energies formed according to the Penrose process. We evaluate in detail the orbital extension of this region and the levels of the energies and angular momentum. This material is obviously formed inside the ergoregion since it cannot penetrate from the external region; the static limit would act indeed as a semi-permeable membrane separating the spacetime region, filled with negative energy particles, from the external one filled with positive energy particles, gathered from infinity or expelled from the ergoregion with impoverishment of the source energy. In any case, although on the equatorial plane the ergoregion is invariant with respect to any transformation involving a change in the source spin (but not with respect to a change in the mass $M$ ), the dynamical structure of the ergoregion is not invariant with respect to a change in the spin-tomass ratio. We will show the presence of limiting geometries where the dynamical structure of the ergoregion outlines a relevant change for a spin shift, where, as a consequence of the interaction of the surrounding matter, one would expect consequently a shift of the source from one class to another. This effect might have relevant consequences as a corollary of the runaway instability from relativistic thick disks, especially for (initial) specific geometries [11-13]. In fact, our analysis enlightens a set of special attractors and particularly the spins $a_{b}^{-} / M \approx 0.828427$ and $a_{2} / M \approx 0.942809$, where a slight change in the attractor spin produces a relevant change in the dynamical structure of the ergoregion, causing in turn a change in the stability properties of the orbital matter, and possibly modifying the closed topology of the orbiting extended matter configuration which passes from an equilibrium state to the accretion and, finally, to an open topology with matter jets.

This paper is organized as follows. In Sect. 2, we present the Kerr line element and briefly discuss the main physical properties of the corresponding geometry. We present the effective potential that governs the dynamics of the test particle motion on the equatorial plane and, in Sect. 2.1, we derive the conditions for the existence and stability of circular orbits. In Sects. 3.1 and 3.2, we explore the relevant orbital regions in the case of black holes and naked singularities. In the appendices we include the definitions of the main radii that determine the dynamical structure of the ergoregion as well as the analysis of the particular limiting cases of extreme black holes and the static boundary. Finally, in Sect. 4, we discuss the results and perspectives of our work.

\section{The Kerr geometry}

The Kerr spacetime is an exact solution of Einstein's equations in vacuum describing an axisymmetric, stationary (nonstatic), asymptotically flat gravitational field. In spheroidal Boyer-Lindquist (BL) coordinates the Kerr line element has the form

$$
\begin{aligned}
\mathrm{d} s^{2}= & -\mathrm{d} t^{2}+\frac{\rho^{2}}{\Delta} \mathrm{d} r^{2}+\rho^{2} \mathrm{~d} \theta^{2}+\left(r^{2}+a^{2}\right) \sin ^{2} \theta \mathrm{d} \phi^{2} \\
& +\frac{2 M}{\rho^{2}} r\left(\mathrm{~d} t-a \sin ^{2} \theta \mathrm{d} \phi\right)^{2},
\end{aligned}
$$

where 
$\Delta \equiv r^{2}-2 M r+a^{2}$, and $\rho^{2} \equiv r^{2}+a^{2} \cos ^{2} \theta$.

Here $M$ and $a$ are arbitrary constants interpreted as the mass and rotation parameters, respectively. The specific angular momentum is $a=J / M$, where $J$ is the total angular momentum of the gravitational source. In this work, we will consider the Kerr black-hole (BH) case defined by $a \in] 0, M[$, the extreme black-hole source $a=M$, and the naked singularity (NS) case where $a>M$. The limiting case $a=0$ is the Schwarzschild solution. The outer and inner ergosurfaces $r_{\varepsilon}^{ \pm}$and Killing (outer and inner) horizons $r_{ \pm}$are determined, respectively, by the equations $g_{t t}=0$, and $g^{r r}=0$ with

$$
\begin{aligned}
& r_{\varepsilon}^{ \pm} \equiv M \pm \sqrt{M^{2}-a^{2} \cos ^{2} \theta}, \quad r_{ \pm} \equiv M \pm \sqrt{M^{2}-a^{2}}, \\
& \text { where } r_{\varepsilon}^{-} \leq r_{-} \leq r_{+} \leq r_{\varepsilon}^{+} .
\end{aligned}
$$

On the equatorial plane $(\theta=\pi / 2)$, it is $\rho=r$ and the spacetime singularity is located at $r=0$ (a curvature ringlike singularity only occurs at $\theta=\pi / 2$ for $M \neq 0$, while generally the metric is singular at $\rho=0$, and the spacetime is clearly flat for $M=0)$. The region $\left.\Sigma_{\varepsilon}^{+} \equiv\right] r_{+}, r_{\varepsilon}^{+}$[ for $a \leq M$ (where $g_{t t}>0$ ) is called ergoregion. In blackhole spacetimes, the $r$-coordinate is spacelike in the intervals $r \in] 0, r_{-}\left[\cup r>r_{+}\right.$, and timelike in the region $\left.r \in\right] r_{-}, r_{+}[$. This means that the surfaces of constant $r$, say ${ }^{1} \Sigma_{r}$, are timelike for $\Delta>0$, spacelike for $\Delta<0$, and null for $\Delta=0$. On the other hand, for $r \in] r_{\varepsilon}^{-}, r_{\varepsilon}^{+}$[ the metric component $g_{t t}$ changes its sign and vanishes for $r=r_{\varepsilon}^{ \pm}$and $\left.\left.\cos ^{2} \theta \in\right] 0,1\right]$, and also at $r=2 M$ for $\theta=\pi / 2$. As the spin of the attractor reaches the limiting value $a=M$ of an extreme $\mathbf{B H}$ source, the horizons coincide, $r_{-}=r_{+}=M$, and if $\cos ^{2} \theta=1$, i.e., on the rotational axis, it is $r_{\varepsilon}^{ \pm}=r_{ \pm}$. On the other hand, in naked singularity geometries, the radii $r_{ \pm}$are not real and the singularity at $\rho=0$ is not covered by a horizon. However, the ergosurfaces $r_{\varepsilon}^{ \pm}$are still well defined. ${ }^{2}$ We can define as well in the equatorial plane the ergoregion $\left.\Sigma_{\varepsilon}^{+} \equiv\right] 0, r_{\varepsilon}^{+}$[ for $a>M$, and $\Sigma_{\varepsilon}^{+}$has a toroidal topology centered on the axis whose inner circle is the naked singularity. In this work, we focus on the dynamics inside the ergoregion at $\theta=\pi / 2$, where $\left.r_{\varepsilon}^{+}\right|_{\pi / 2}=\left.r_{+}\right|_{a=0}=2 M$ and $r_{\varepsilon}^{-}=0$, and we compare the dynamical properties of circularly orbiting matter in $\Sigma_{\varepsilon}^{+}$for the $\mathbf{B H}$ and $\mathbf{N S}$ cases.

The motion of matter in $\Sigma_{\varepsilon}^{+}$has very peculiar properties. We summarize here some of the major characteristics relevant for this analysis. First, the equatorial (circular) trajectories are confined in the equatorial geodesic plane as a consequence of the metric tensor symmetry under reflection through the equatorial hyperplane $\theta=\pi / 2$. A fundamental property of the ergoregion from the point of view of the

\footnotetext{
$\overline{{ }^{1} \Sigma_{\mathbf{Q}} \text { is the } \mathbf{Q}}=$ constant surface for any quantity or set of quantities Q.

${ }^{2}$ In particular, we have $g_{t t}<0$ in $0 \leq \cos \theta^{2} \leq M^{2} / a^{2}$ for $r \in$ ] $0, r_{\varepsilon}^{-}\left[\cup r>r_{\varepsilon}^{+}\right.$and in $\left.\left.\cos \theta^{2} \in\right] M^{2} / a^{2}, 1\right]$ for $r>0$.
}

matter and field dynamics is that no matter can be at rest in $\Sigma_{\varepsilon}^{+}$(as seen by a faraway observer or, in other words, from infinity in a BL coordinate frame) [40]. Then the metric is no longer stationary. As we have already noted, in the BL coordinates the surfaces of constant $(r, \theta, \phi)$, with line element $\left.\mathrm{d} s\right|_{\Sigma_{r, \theta, \phi}}$, are spacelike inside the ergoregion, that is, the "time" interval becomes spacelike, and in terms of BL coordinates this means that $t$ is spacelike and any motion projected into $\Sigma_{r, \theta, \phi}$ is forbidden. The outer ergosurface $r_{\varepsilon}^{+}$is called the static surface or static limit (also stationary limit surface; see [41]). This is a timelike surface, except on the axis of a Kerr source, where it matches the outer horizon; then it is nullike. At $r>r_{\varepsilon}^{+}$particles can follow an orbit of the vector $\xi_{t}$ and eventually cross the static limit (away from the axis). Moreover, for a timelike particle (with positive energy) it is possible to cross the static limit and to escape toward infinity. In the static $\mathbf{B H}$ spacetime $(a=0)$, the region $] 0,\left.r_{+}\right|_{a=0}[$ coincides with the zone inside the horizon; then no particle can stay at rest (with respect to an observer located at infinity) neither at $r=$ constant, i.e., any particle is forced to fall down into the singularity. For the stationary spacetimes $(a \neq 0)$ in $\Sigma_{\varepsilon}^{+}$the motion with $\phi=$ const is not possible, and all particles are forced to rotate with the source i.e. $\dot{\phi} a>0$. Indeed, in the ergoregion the Killing vector $\xi_{t}^{a}=(1,0,0,0)$ becomes spacelike, i.e., $g_{a b} \xi_{t}^{a} \xi_{t}^{b}=g_{t t}>0$. This fact implies in particular that a static observer, i.e. an observer with fourvelocity proportional to $\xi_{t}^{a}$ so that $\dot{\theta}=\dot{r}=\dot{\phi}=0$ (the dot denotes the derivative with respect to the proper time $\tau$ along the curve) cannot exist inside the ergoregion. Therefore for any in-falling matter (timelike or photonlike) approaching the horizon $r_{+}$, in the region $\Sigma_{\varepsilon}^{+}$, we have $t \rightarrow \infty$ and $\phi \rightarrow \infty$, meaning that the world-lines around the horizon, as long as $a \neq 0$, are subject to an infinite twisting. Trajectories with $r=$ const and $\dot{r}>0$ (particles crossing the static limit and escaping outside to $r \geq r_{\varepsilon}^{+}$) are possible. Another important point is that for an observer at infinity, the particle will reach and penetrate the surface $r=r_{\varepsilon}^{+}$, in general, in a finite time $t$. For this reason, the ergoregion boundary is not a surface of infinite redshift, except for the axis of rotation where the ergoregion coincides with the event horizon. Indeed, concerning the frequency of a signal emitted by a source in motion along the boundary of the ergoregion $r_{\varepsilon}^{+}$, it is clear that the proper time of the source particle is not null. ${ }^{3}$ This means that the observer at infinity will see a non-zero emission frequency. In the spherical symmetric case $(a=0)$, however, as $g_{t \phi}=0$ the proper time interval $\mathrm{d} \tau=\sqrt{\left|g_{t t}\right|} \mathrm{d} t$, goes to zero as one approaches $r=r_{+}=r_{\varepsilon}^{+}$. Thus, in the equatorial plane as $a \rightarrow 0$ and the geometry "smoothly" resembles the spherical symmetric case the frequency of the

\footnotetext{
3 However, as we have $g_{t t}\left(r_{\varepsilon}^{ \pm}\right)=0$, it is also named infinity redshift surface; see for example [41].
} 
emitted signals, as seen by an observer at infinity, goes to zero.

\subsection{On the particle's energy and the effective potential}

In this section, we discuss some aspects of particle energy definition and the effective potential for the circular orbits, which will be used here to analyze the dynamics inside the ergoregion $\Sigma_{\varepsilon}^{+}$. Let $u^{a}=\mathrm{d} x^{a} / \mathrm{d} \tau=\dot{x}^{a}$ be the tangent vector to a curve $x^{a}(\tau)$, the momentum $p^{a}=\mu \dot{x}^{a}$ of a particle with mass $\mu$ being normalized so that $g_{a b} \dot{x}^{a} \dot{x}^{b}=-k$, where $k=$ $0,-1,1$ for null, spacelike, and timelike curves, respectively. In order to simplify the investigation of the circular dynamics we use the symmetries of the Kerr geometry: since the metric tensor is independent of $\phi$ and $t$, the covariant components $p_{\phi}$ and $p_{t}$ of the particle's four-momentum are conserved along its geodesic, i.e., the quantities

$$
\begin{aligned}
& E \equiv-g_{a b} \xi_{t}^{a} p^{b}=-\left(g_{t t} p^{t}+g_{t \phi} p^{\phi}\right), \\
& L \equiv g_{a b} \xi_{\phi}^{a} p^{b}=g_{\phi \phi} p^{\phi}+g_{t \phi} p^{t}
\end{aligned}
$$

are constants of motion, where $\xi_{\phi}=\partial_{\phi}$ is the rotational Killing field and $L$ is interpreted as the angular momentum of the particle as measured by an observer at infinity. The Killing field $\xi_{t}=\partial_{t}$ represents the stationarity of the spacetime and we may interpret $E$, for timelike geodesics, as the total energy of the test particle coming from radial infinity, as measured by a static observer located at infinity.

An essential characteristic of the region we are investigating is to foreseen negative energy states for the matter dynamics, therefore it is convenient here to give a more detailed definition of the energy. In general, the particle's energy could be defined as $E_{\tau}$ in terms of $\partial \mathscr{S} / \partial \tau$ or as $E_{t} \equiv E$ in terms of $\partial \mathscr{S} / \partial t$, where $\mathscr{S}$ is the particle action. The energy $E_{\tau}$ is defined with respect to the proper time of the particle synchronized along the trajectory. This quantity is always positive, but in general it is not conserved. On the other hand, the definition of $E_{t}$ contains the derivative with respect to the universal time, taking account of the symmetries of the stationary spacetime, and consequently it is constant along the orbit of the timelike Killing vector. This quantity, as defined in Eq. (4), is conserved, but it can be negative in $\Sigma_{\varepsilon}^{+}$, where $t$ is no more a timelike coordinate [40]. The particle with negative energy cannot escape to infinity, but its dynamics is confined in $r<r_{\varepsilon}^{+}$. Therefore the static limit would act as a semi-permeable membrane separating the spacetime region $\Sigma_{\varepsilon}^{+}$, filled with negative energy particles, from the external one filled with particles with positive energy, gathered from infinity or expelled from the ergoregion with the consequent impoverishment of source energy. However, in the case of a naked singularity this phenomenon is combined with the repulsive effects from the singularity ring $r=0$, and consequently the region $\Sigma_{\varepsilon}^{+}$will have a more articulated structure as regards the matter dynamics. Possibly, as pointed out in [35], a set of negative energy particles will form (from the Penrose process) in $\Sigma_{\varepsilon}^{+}$, so one task is to study what the fate is of the negative energy particles and what role these particles play in the evolution of the source. The relevance of the speculated Penrose process in the eventual formation in $\Sigma_{\varepsilon}^{+}$of an extended configuration of negative energy matter cannot be ruled by considerations of the geometric properties of spacetime only. However, in this work we address the question of what would happen to the particle dynamics in $\Sigma_{\varepsilon}^{+}$as well as to the dynamics of the extended object under hydrostatic pressure, focusing in particular on the energetic properties of the test particles and on the necessary conditions for the formation and stability of toroidal, pressure supported accretion disks. As we focus here on the specific case of circular motion, one could ask if the circular motion (stable or not) of particles with negative energy is possible for some kind of sources.

We start our analysis noting that, using the definitions given in Eq. (4), the investigation of the circular motion of test particles in the equatorial plane can be reduced to the study of the motion in the effective potential $V$, defined from the normalization condition of the particle's four-velocity

$g_{t t} \dot{t}^{2}+g_{\phi \phi} \dot{\phi}^{2}+2 g_{\phi t} \dot{t} \dot{\phi}+g_{r r} \dot{r}^{2}=-k$,

where the condition $\dot{\theta}=0$ has been used as geodesics starting in the equatorial plane are planar. Using Eq. (4) for a particle in circular motion, i.e. $\dot{r}=0$, we obtain from Eq. (5) the effective potential

$V^{ \pm}=\frac{-g_{\phi t} L \pm \sqrt{\left(g_{\phi t}^{2}-g_{t t} g_{\phi \phi}\right)\left(L^{2}+g_{\phi \phi} k \mu^{2}\right)}}{g_{\phi \phi}}$,

which represents the value of $E / \mu$ that makes $r$ into a "turning point" ( $V=E / \mu)$; in other words, it is the value of $E / \mu$ at which the (radial) kinetic energy of the particle vanishes. The (positive) effective potential can be written explicitly as

$V \equiv \frac{V^{+}}{\mu}=-\frac{\beta}{2 \alpha}+\frac{\sqrt{\beta^{2}-4 \alpha \gamma}}{2 \alpha}$

where $[42,43]$

$\alpha \equiv\left(r^{2}+a^{2}\right)^{2}-a^{2} \Delta, \quad \beta \equiv-2 a L\left(r^{2}+a^{2}-\Delta\right)$,

$\gamma \equiv a^{2} L^{2}-\left(M^{2} r^{2}+L^{2}\right) \Delta$.

The behavior of the effective potential $V^{-}$can be studied by using the following symmetry $V^{+}(L)=-V^{-}(-L)$. The Kerr metric (1) is invariant under the application of any two different transformations: $\mathscr{P}_{\mathbf{Q}}: \mathbf{Q} \rightarrow-\mathbf{Q}$, where $\mathbf{Q}$ is one of the coordinates $(t, \phi)$ or the metric parameter $a$, a single transformation leads to a spacetime with an 
opposite rotation respect to the unchanged metric. Thus, we fix $a \geq 0$ and we note that the potential function (7) is invariant under the mutual transformation of the parameters $(a, L) \rightarrow(-a,-L)$. We will restrict the analysis to the case of positive values of $a$ for corotating $(L>0)$ and counterrotating $(L<0)$ orbits. For (timelike) circular orbits we have

$\dot{r}=0, \quad V=\frac{E}{\mu}, \quad \frac{\mathrm{d} V}{\mathrm{~d} r}=V^{\prime}=0$

(see also [19]). Solving the equation $V^{\prime}=0$ with respect to the angular momentum, we find

$$
\frac{L_{ \pm}}{\mu M} \equiv \frac{\left|\frac{a^{2}}{M^{2}} \pm 2 \frac{a}{M} \sqrt{\frac{r}{M}}+\frac{r^{2}}{M^{2}}\right|}{\sqrt{\frac{r^{2}}{M^{2}}\left(\frac{r}{M}-3\right) \mp 2 \frac{a}{M} \sqrt{\frac{r^{3}}{M^{3}}}}} .
$$

Any particle moving along a circular orbit in these spacetimes has an angular momentum of a magnitude of either $L_{+} \geq$ 0 or $L_{-} \geq 0$. Introducing Eq. (10) into (7), we find the energies $E_{ \pm} \equiv E\left(L_{ \pm}\right)$and $E_{ \pm}^{-} \equiv E\left(-L_{ \pm}\right)$. The orbits with angular momentum $L=\mp L_{ \pm}$are allowed in different orbital regions for different attractors, depending on the corotating or counterrotating nature of the motion.

The energies $\mathscr{E} \equiv\left\{E_{ \pm}, E_{ \pm}^{-}\right\}$and the angular momenta $\mathscr{L} \equiv\left\{L_{ \pm},-L_{ \pm}\right\}$are functions of the spacetime spin-mass ratio and, therefore, we use them in this work to characterize different attractors with different spin-mass ratios. In general, depending on the context, by $\mathscr{E}$ or $\mathscr{L}$ we shall mean, if not otherwise specified, either the set of the respective quantities or a generic element of the set, which will be specified when necessary. The boundaries of these regions are determined by a set $r_{i} \in \mathscr{R}$, different for $\mathbf{B H}$ and NS configurations, and include the radii $\left\{r_{\gamma}, r_{b}, r_{l s c o}\right\}$ as given in Table 1. These radii are defined through different expressions for corotating $(-)$ and counterrotating orbits $(+)$ in a naked singularity or in black-hole geometries, respectively (see also Appendix A). The physical importance of these radii can be explained as follows: Timelike circular orbits can fill the spacetime region $r>r_{\gamma}$, and the orbits at $r_{\gamma}$, as defined in Eq. (A.1), are photon orbits (lightlike orbits), known as marginally circular orbits or also last circular orbits. It should be noted, however, that orbits with radius $r_{\gamma}$ are not timelike, but they are limiting values for timelike free particles and matter where the angular momentum diverges. Thus no circularly orbiting (timelike) matter can be formed in the region $\left.\Sigma_{\varnothing} \equiv\right] r_{+} r_{\gamma}$, because the energy and angular momentum of the particle diverge as the photonlike orbit is approached.

Stable orbits are in the region $\left.\Sigma_{s} \equiv\right] r_{l s c o},+\infty[$, and the orbits at $r_{l s c o}$, defined in Eqs. (A.2) and (A.3), are named, accordingly, marginally stable circular orbits or also, quite improperly, last stable circular orbits (see, for example, [44, 45]), respectively, for $\mathbf{B H}$ and NS sources and for different types of orbits, as specified in Appendix A. While it should be kept in mind that $r_{l s c o}$ does not correspond to a stable orbit, as it is indeed a saddle point for the effective potential, that is, $\left.d_{r} V\right|_{r_{l s c o}}=\left.d_{r}^{2} V\right|_{r_{l s c o}}=0$, the marginally stable orbits correspond to the minimum of the energy and angular momentum of the particle [19]).

The instability region $\left.\left.\Sigma_{u} \equiv\right] r_{\gamma}, r_{l s c o}\right]$ is split by the radius $r_{b}$, where $\mathscr{E}\left(r_{b}\right)=1$, generally into two regions $\Sigma_{u}=$ $\Sigma_{u}^{\geq} \cup \Sigma_{u}^{<}$where $r_{b}$, defined in Eqs. (A.5) and (A.6), determines the marginally bounded orbit or the last bounded orbit. For $\left.\left.\Sigma_{\bar{u}}^{\geq} \equiv\right] r_{\gamma}, r_{b}\right]$ we have $\mathscr{E} \geq 1$ and for $\left.\left.\Sigma_{u}^{<} \equiv\right] r_{b}, r_{l s c o}\right]$ we have $\mathscr{E}<1$. In Sect. 3.2.1, we will discuss the special case where the energy parameter can be negative. The explicit expressions of the radii $\mathscr{R}$ for black holes are given, for example, in [19]. In this work, we focus on the case of naked singularities, specifying the exact form of $r_{i} \in \mathscr{R}$ for different classes of super-spinning objects $(a>M)$.

The parameters $\mathbf{p} \equiv\{\mathscr{L}, \mathscr{E}\}$, and the radii $\mathscr{R}$ determine the main properties of test particles moving along circular orbits. These are the main quantities that will be used below to explore the physical properties of spacetimes described by the Kerr metric. We investigate the dynamics in the ergoregion $\Sigma_{\varepsilon}^{+}$through the study of the regions $\Sigma \equiv\left\{\Sigma_{s}, \Sigma_{u}, \Sigma_{\varnothing}\right\}$, which are characterized by the geometric properties $\Sigma_{\varepsilon}^{+}=\bigcup \Sigma_{i}$ and $\Sigma_{j} \bigcap \Sigma_{i}=\emptyset, \forall i, j$. The dynamical structure of the ergoregion is important because it fully characterizes the dynamical properties of the orbiting matter inside $\Sigma_{\varepsilon}^{+}$.

The properties of matter in circular configurations, as well as toroidal configurations of extended matter in accretion disks, are in general governed by several factors such as the pressure, the magnetic fields, the dissipative effects and so on; however, the properties of the spacetime structure, regulated by the radii $\mathscr{R}$, are the basis on which to build any disk model [32], especially in thick pressure supported relativistic accretion disks where the background curvature strongly affects the equilibrium and the disk instability. The disk equatorial plane of symmetry is often aligned with the symmetric plane of the accretor. By using the symmetry of the system, the geometric symmetries of the background and of the matter configuration, we are capable to reduce the analysis to an one-dimensional problem along the radial direction $r$, the dynamics of the orbiting matter being regulated entirely by the couple of model parameters $\mathbf{p}$, which are constant along the geodesics because they are associated with the orbits of the Killing vector fields. Then the main features of the disk dynamics, stability and accretion, are mainly regulated by the properties of the disk as projected on the plane of symmetry $\theta=\pi / 2$. Moreover, the region $\Sigma_{\varepsilon}^{+}$is closely involved in the evolutionary processes of the accretor, resulting in a shift of the intrinsic spin. As a consequence of the interaction of the surrounding matter in $\Sigma_{\varepsilon}^{+}$, one would expect a change of the source spin, resulting in a change of the corresponding class 
Table 1 Set of spins $\mathscr{A}$ and radii $\mathscr{R}$ for the black-hole $(\mathbf{B H})$ and the naked singularity (NS) geometries, respectively. The radii $\mathscr{R}_{B H}=$ $\left\{r_{\gamma}^{-}, r_{b}^{-}, r_{l s c o}^{-}\right\}$set the photon orbit (or also last circular orbit), the marginally bounded orbit, and the last stable circular orbit, respectively (for corotating orbits) in black-hole geometries. See also Figs. 1 and 4, and the discussions in Sect. 3.1 for the BH case, and Sect. 3.2 for the NS case. For the naked singularity case the radii $\mathscr{R}_{N S}=$ $\left\{r_{v}^{ \pm}, \hat{r}_{ \pm}, r_{l s c o}^{(N S)-}, r_{b}^{(N S)}\right\}$ set the outer $\left(r_{v}^{+}\right)$and inner $\left(r_{v}^{-}\right)$effective ergosurfaces, where the orbital energies $E=0$ (see Sect. 3.2), and the radii $\hat{r}_{ \pm}$(zero angular momentum radii), where $\mathscr{L}\left(\hat{r}_{ \pm}\right)=0$. Moreover, $r_{b}^{(N S)}$ and $r_{l s c o}^{(N S)-}$ are the marginally bounded orbit and the last stable circular orbit, respectively, for the NS geometries. The explicit expression of the radii can be found in Appendix A

Black-hole classes: $\mathscr{A}=\mathscr{A}_{B H} \equiv\left\{0, a_{1}, a_{b}^{-}, a_{2}, M\right\}, \mathscr{R}=\mathscr{R}_{B H} \equiv\left\{r_{\gamma}^{-}, r_{b}^{-}, r_{l s c o}^{-}\right\}$

$a_{1} / M \equiv 1 / \sqrt{2} \approx 0.707107 ; \quad a_{b}^{-} / M \equiv 2(\sqrt{2}-1) \approx 0.828427 ; \quad a_{2} / M \equiv 2 \sqrt{2} / 3 \approx 0.942809$

$a_{1}: r_{\gamma}^{-}\left(a_{1}\right)=r_{\varepsilon}^{+} ; \quad a_{b}^{-}: r_{b}^{-}\left(a_{b}^{-}\right)=r_{\varepsilon}^{+} ; \quad a_{2}: r_{l s c o}^{-}\left(a_{2}\right)=r_{\varepsilon}^{+}$

BHI : $\left[0, a_{1}\left[\right.\right.$ BHIIa : $\left[a_{1}, a_{b}^{-}\left[; \quad\right.\right.$ BHIIb : $\left[a_{b}^{-}, a_{2}\left[; \quad\right.\right.$ BHIII : $\left[a_{2}, M\right]$

Naked singularity classes: $\mathscr{A}=\mathscr{A}_{N S} \equiv\left\{a_{\mu}, a_{3}, a_{4}, a_{b}^{N S}\right\}, \mathscr{R}=\mathscr{R}_{N S} \equiv\left\{r_{v}^{ \pm}, \hat{r}_{ \pm}, r_{l s c o}^{(N S)-}, r_{b}^{(N S)}\right\}$

$a_{\mu} / M=4 \sqrt{2 / 3} / 3 \approx 1.08866 ; \quad a_{3} / M \equiv 3 \sqrt{3} / 4 \approx 1.29904 ; \quad a_{4} / M \equiv 2 \sqrt{2} \approx 2.82843: \quad a_{b}^{N S} / M \approx 4.82843$

$a_{\mu}: r_{v}^{+}\left(a_{\mu}\right)=r_{v}^{-}\left(a_{\mu}\right) ; \quad a_{3}: \hat{r}_{+}\left(a_{3}\right)=\hat{r}_{-}\left(a_{3}\right) ; \quad a_{4}: r_{l s c o}^{(N S)-}\left(a_{4}\right)=r_{\varepsilon}^{+} ; \quad a_{b}^{N S}: r_{b}^{(N S)}\left(a_{b}^{N S}\right)=r_{\varepsilon}^{+}$

NSIa : ] $\left.M, a_{\mu}\right]$; NSIb : $\left.] a_{\mu}, a_{3}\right] ; \quad$ NSII : $\left.] a_{3}, a_{4}\right] ; \quad$ NSIIIa : $\left.] a_{4}, a_{b}^{N S}\right] ; \quad$ NSIIIlb : $\left.] a_{b}^{N S},+\infty\right]$

or subclass of the attractor, with potentially relevant phenomenological implications [11-13]. Furthermore, in [35] it is argued that the ergoregion (in any plane $\theta$ ) cannot disappear as a consequence of a spin shift as it is supposed to be filled by the negative energy matter; in fact, the limiting case corresponds to $r_{+}=r_{\varepsilon}^{+}=2 M$. On the equatorial plane, the ergoregion (for $a \neq 0$ ) is invariant with respect to any transformation involving a change in the source spin (but not with respect to a change in the mass $M$ ), but the dynamical structure of $\Sigma_{\varepsilon}^{+}$is not invariant under changes of the ratio $a / M$; in other words, the limit $r_{\varepsilon}^{+} /\left.M\right|_{\theta=\pi / 2}=2$ is independent of the central attractor, but not of its dynamical structure $\Sigma$. We will show the presence of limiting geometries from the point of view of the structure of $\Sigma_{\varepsilon}^{+}$, where the dynamical structure of the ergoregion can change significantly as a consequence of a small shift of the attractor spin (see also Appendix B).

\section{Analysis and discussion of the dynamics}

In this section, we discuss the particle dynamics circularly orbiting in $\Sigma_{\varepsilon}^{+}$, and characterizing the different attractors in terms of the dynamical structure $\Sigma$ of the ergoregion. The case of BH geometries is addressed in Sect. 3.1, and the NS case is considered in Sect. 3.2. A similar analysis for the region $r>r_{\varepsilon}^{+}$was discussed in [19]. Our results lead to the identification of different classes of attractors, defined by the spin-mass ratios in the regions of the $r-a$ plane with boundaries in $\mathscr{R} \otimes \mathscr{A}$, where $\mathscr{A}$ is the set of spins defined at the crossings of the radii $\left(r_{i}, r_{j}\right)$ in $\mathscr{R}$ and $r_{\varepsilon}^{+}$in $\Sigma_{\varepsilon}^{+}$(see Table 1 ). We identify four classes of blackhole sources $(a \leq M)$ and five classes of naked singularities $(a>M)$, defined in Table 1 . The classes of geometries have boundaries in $\mathscr{A}=\mathscr{A}_{B H}$ and $\mathscr{R}=\mathscr{R}_{B H}$ for black holes, and
$\mathscr{A}=\mathscr{A}_{N S}$ and $\mathscr{R}=\mathscr{R}_{N S}$ for naked singularities (details are given in Table 1). The geometries with spin $a_{i} \in \mathscr{A}_{B H} \cup$ $\mathscr{A}_{N S}$ are limiting cases with respect to the structure of $\Sigma_{\varepsilon}^{+}$. Particularly relevant is the extreme case $a=M$, which marks also the limit between black holes and naked singularities; this case will be addressed in particular in Appendix B. Close to the boundary spacetimes, in particular, as discussed in Appendix $\mathrm{B}$, at $a=a_{2} \approx 0.942809 M$ and $a=M$, the properties around the limiting spin value are rather subject to a sort of fine-tuning, i.e. at a fixed radial distance from the source, the dynamical properties modify significantly as consequence of a slight change in the spin (see Appendix B). For this reason, the geometries with the spin in $\mathscr{A}$ are likely to give rise to rather relevant phenomena for the geometrical properties of the matter configurations orbiting in regions very close to the source; see also Appendix B.

Photonlike circular orbits in $\Sigma_{\varepsilon}^{+}$are a feature of BHII and BHIII sources only; there are no photonlike orbits inside the ergoregion $\Sigma_{\varepsilon}^{+}$in NS spacetimes. In NS spacetimes, there is no last circular orbit for corotating particles with $L=L_{-}$, indicating that circular orbits can theoretically be up to the singularity. In other words, in this case there is at least one circular orbit for any $r>0$ as long as the particle's angular momentum assumes certain values. On the other hand, counterrotating orbits $\left(L=-L_{-}\right)$, characteristic of NSI sources, can exist only in a bounded orbital region. Stability regions $\Sigma_{s} \subset \Sigma_{\varepsilon}^{+}$are present only in the classes BHIII, NSI, and NSII (see Table 1; Figs. 1 and 4).

We will investigate the structure $\Sigma$ of $\Sigma_{\varepsilon}^{+}$, providing a characterization of the regions of $\Sigma$ and, in particular, the length or extension of each orbital region in which $\Sigma_{\varepsilon}^{+}$is decomposed, where the extension $\mu_{\varepsilon}^{+}=2 M$ of $\Sigma_{\varepsilon}^{+}$is the sum of the lengths of all regions of $\Sigma$. To understand the physical implications of this analysis, we will focus our discussion on two different orbiting configurations: the case of 

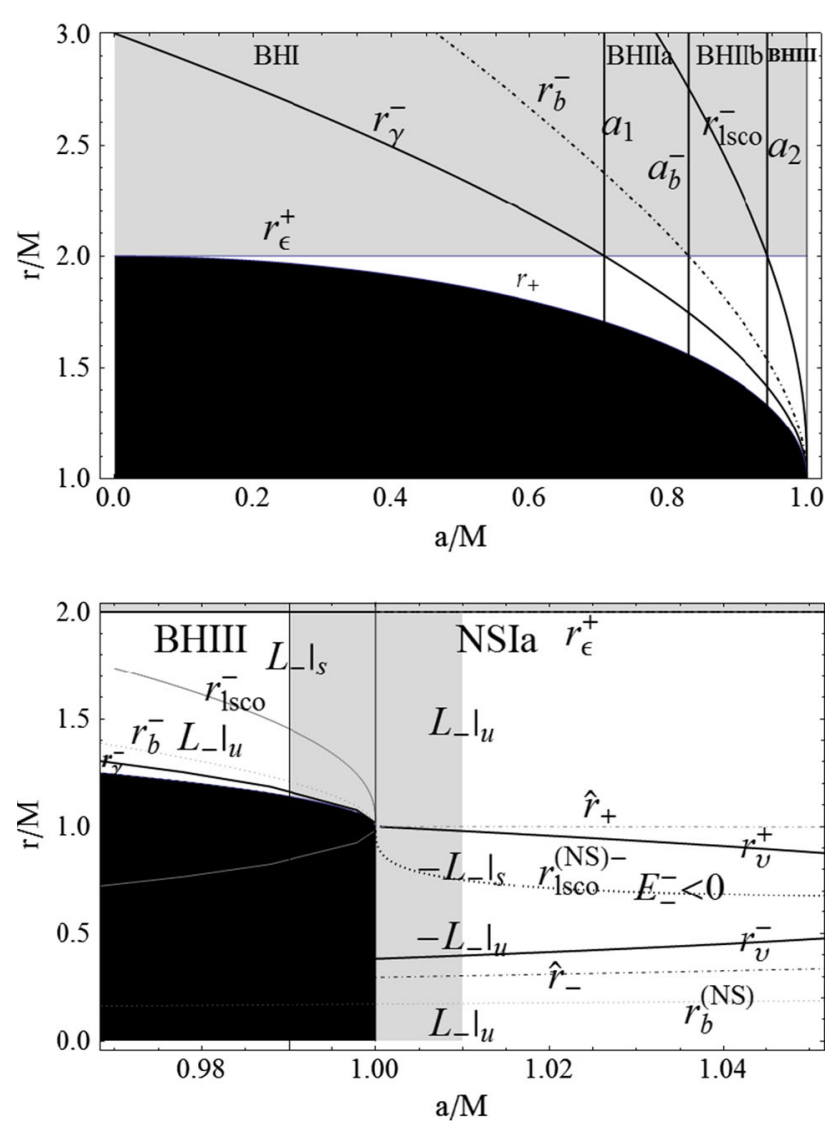

Fig. 1 Arrangement of the radii $r_{i} \in \mathscr{R}_{B H} \cup \mathscr{R}_{N S}$ determining the properties of circular orbits around a rotating central mass and classes of attractors for black-hole (BH) (upper and bottom panels) and naked singularity (NS) sources (bottom panel), as given in Table 1. $\left(r_{b}^{-}, r_{b}^{(N S)}\right.$ ) are the marginally bounded orbits, $\left(r_{l s c o}^{-}, r_{l s c o}^{(N S)-}\right)$ are the marginally stable orbits, $r_{\gamma}^{-}$is the photon (last circular) orbit. The region outside the static limit $r>r_{\varepsilon}^{+}$is in gray, and the one with $r<r_{+}$is in black. The bottom panel shows in light gray a neighborhood of the geometry $a=M$ between the BHIII and NSIa classes. The orbital stability in each region is analyzed and explicitly shown: the angular momentum is quoted with its stability property as $\left.\right|_{s}$ for stable and $\left.\right|_{u}$ for unstable orbits. Radii $\hat{r}_{ \pm}$are zero angular momentum orbits $(L=0)$, and the radii $r_{v}^{ \pm}$, zero energy circular orbits, are counterrotating $\left(L=-L_{-}\right)$ orbits with $E=0$. The region $] r_{v}^{-}, r_{v}^{+}$[ contains only counterrotating orbits with $E<0$

Keplerian (dust) disks and the pressure supported accretion thick disk models [15,32,34,38,46,47]. We discuss the physical meaning of the regions $\Sigma_{s} \Sigma_{u}$ and $\Sigma_{\varnothing}$ in relation to these astrophysical models.

The region $\Sigma_{s}$ consists of stable geodesic orbits and, in the case of a dust disk, the length $\mu_{s}$ of $\Sigma_{s}$ indicates the amount of dust that can be in equilibrium inside the ergoregion $\Sigma_{\varepsilon}^{+}$. According to the current accretion disk models, the upper bound for the inner edge of the disk is actually located in a region $r \leq r_{l s c o}$; then the length $\mu_{s}$ of the orbital region $\Sigma_{s} \subseteq \Sigma_{\varepsilon}^{+}$provides the maximum elongation, in the equatorial plane inside the ergoregion, of a hypothetical accre- tion disk with inner edge in $\Sigma_{\varepsilon}^{+}$. Then the disk penetrates the ergoregion, crossing the static limit (see, for example, [34]), or it is totally included in $\Sigma_{\varepsilon}^{+}$. If the disk is thick, one can use the Boyer model (see [48]) for a pressure supported thick accretion disk, where the center of the configuration $r_{c}$, which locates the maximum of the hydrostatic pressure, is inside the stable region, i.e., $r_{c} \in \Sigma_{s} \subseteq \Sigma_{\varepsilon}^{+}$. However, the inner edge $r_{\mathscr{K}}^{i}$ of the disk, in the case of a thick disk in equilibrium as established by the PaczynskiWiita $(\mathrm{P}-\mathrm{W})$ mechanism [32], can also be located in a region $r_{b}<r_{\max }<r_{\mathscr{K}}^{i}<r_{l s c o}<r_{\min }<r_{\mathscr{K}}^{o}$, where $r_{\mathscr{K}}^{o}$ denotes the outer edge and $\left(r_{\max }, r_{\min }\right)$ are the maximum and minimum points of the effective potential, respectively.

The instability region $\Sigma_{u}$, filled with unstable circular orbits, is generally split into two regions (which may be connected or not) as $\Sigma_{u}=\Sigma_{\bar{u}}^{\geq} \cup \Sigma_{u}^{<}$. In the instability region, there could be a decay phenomenon in which a particle may either escape, spiraling into the outer region and therefore possibly becoming observable, or be captured by the source, changing its spin-to-mass ratio (see, for example, [42]). This phenomenon, in turn, could also may give rise to jets of matter. The instability region has indeed an important role for the relativistic thick accretion disk model as it is essential for the modeling of associated non-equilibrium phenomena related either to the accretion or to the jet production. When the inner edge of the disk is in $r_{\mathscr{K}}^{i} \in \Sigma_{u}^{<}$(on a maximum point of the effective potential) while $r_{c} \in \Sigma_{S}$, this can give rise to a limiting situation, and then $r_{\mathscr{K}}^{i}$ represents an unstable point, a cusp in which matter grows on the attractor, or, as also occurs in some geometries, a point of excretion outwardly. In any case, the material presents a lobe closed to and centered around the maximum pressure point $r_{c}$, while the cusp point represents the overflow point of the material. Then the length $\mu_{s}+\mu_{u}^{<}$, where $\mu_{u}^{<}$is the length of $\Sigma_{u}^{<}$, provides the maximum elongation in the equatorial plane of the distance $r_{c}-r_{\mathscr{K}}^{i}$ for an accretion disk in $\Sigma_{\varepsilon}^{+}$. If the disk is thick, then the density is constant and one could evaluate, in terms of the length $\mu_{s}+\mu_{u}^{<}$, the total mass contained in the configuration with maximum elongation. In $\Sigma_{u}^{\geq}$, where $\mathscr{E} \geq 1$, circular geodesics are unstable and unbounded. According to the Boyer model [48], the extended matter configurations with a minimum point of pressure located in $\Sigma_{\bar{u}}^{\geq}$can open in jets with funnels along the axis. More precisely, the maximum extension $\lambda$ for a pressure supported thick accretion disk (see, for example, [32]) is the elongation $\lambda_{x}$ in the equatorial plane of its critical configuration, i.e., in accretion. Then the inner edge $r_{\mathscr{K}}^{i}$ is located exactly at the maximum of the effective potential and precisely $r_{\mathscr{K}}^{i}=r_{\max } \in \Sigma_{u}^{<}$ and the outer edge is also uniquely fixed by $r_{\max }$. For sufficiently large angular momenta, such that the disk is entirely included in $\Sigma_{\varepsilon}^{+}$, then the upper bound of its elongation $\lambda \equiv r_{\mathscr{K}}^{o}-r_{\mathscr{K}}^{i}$ is $\sup \lambda_{x}=r_{\mathscr{K}_{x}}^{o}-r_{\mathscr{K}_{x}}^{i} \approx \mu_{u}^{<}+r_{\varepsilon}^{+}-r_{b}$ 
Table 2 Dynamical structure of the ergoregion $\Sigma_{\varepsilon}^{+}$and length $\mu$ of its sections in the black-hole geometries. The label ()$_{\varnothing}$ refers to the region where circular orbits are not allowed, ()$_{s}$ refers to the regions where stable circular orbits are possible, ()$_{u}^{<}$is for regions where the orbits are unstable with energy $E<1$ (in units of the mass particle), and finally ()$_{u}^{\geq}$is for the regions where unstable circular orbits are with $E>1$. The corresponding length is denoted by $\mu_{()}$. The extremes of the ranges for the lengths $\mu_{()}$are evaluated for the extreme geometries of each spacetime class. The regions are ordered according to the decreasing orbital distance from the source, that is, from the more distant from the source to the closest. The lengths are in units of mass $M$. See also Fig. 1

Black-hole geometries:

BHI: $\Sigma_{\varepsilon}^{+}=\Sigma_{\varnothing}$

Length of the sections: $\mu_{\varepsilon}^{+}=2 M$

BHIIa: $\Sigma_{\varepsilon}^{+}=\Sigma_{u}^{\geq}\left(L_{-}\right) \cup \Sigma_{\varnothing}$

Length of the sections: $\mu_{u}^{\geq} \in[0,0.253841]$

BHIIb: $\Sigma_{\varepsilon}^{+}=\Sigma_{u}^{<}\left(L_{-}\right) \cup \Sigma_{\bar{u}}^{\geq}\left(L_{-}\right) \cup \Sigma_{\varnothing}$

Length of the sections: $\mu_{u}^{<} \in[0,0.464516], \mu_{u}^{\geq} \in[0.253841,0.120867], \mu_{\varnothing} \in[0.771974,0.417721]$

BHIII: $\Sigma_{\varepsilon}^{+}=\Sigma_{s}\left(L_{-}\right) \cup \Sigma_{u}^{\geq}\left(L_{-}\right) \cup \Sigma_{u}^{<}\left(L_{-}\right) \cup \Sigma_{\varnothing}$

Length of the sections: $\mu_{s} \in[0,1], \mu_{u}^{<} \in[0.464516,0], \mu_{u}^{>} \in[0.120867,0], \mu_{\varnothing} \in[0.417721,0]$

(this is because we are considering in this work the region $r<r_{\varepsilon}^{+}$only and $r_{l s c o}>r_{b}>r_{\gamma}$; we could equivalently say that $\sup \lambda=\mu_{u}^{<}+\mu_{s}$ ). The elongation of the disk in equilibrium, centered in $r_{c}=r_{\text {min }}$, must be lower than this critical value and its inner and outer edges are included in the range ]$r_{\mathscr{K}}^{i}, r_{\mathscr{K}}^{o}[\subset] r_{\mathscr{K}_{x}}^{i}, r_{\mathscr{K}_{x}}^{o}$ [. Then, for example, in BHIII spacetimes we have $\sup \lambda \leq M$ (recall that in BHIII spacetime we have $\partial_{a} \mu_{s}>0$ ). However, even if the evaluation of $\lambda$ depends on the adopted angular momentum, for the equilibrium configuration we have $r_{\max }<r_{l s c o}<r_{\min }=r_{c}<r_{\mathscr{K}}^{o}$, while for the accretion configuration we have $r_{\max }=r_{\mathscr{K}_{x}}^{i}<$ $r_{l s c o}<r_{\min }<r_{\mathscr{K}}^{o}<r_{\mathscr{K}_{x}}^{o}$, with $r_{\mathscr{K}_{x}}^{i}<r_{\mathscr{K}}^{i}$; thus, for instance, in the case of BHIIb sources, where the accretion of even jets can occur, the supremum of the distance $r_{c}-r_{\mathscr{K}_{x}}^{i}$ is $\sup \mu_{u}^{<}$.

In general, one might look at the entire evolution of a thick accretion disk, from the formation of a thin ring, its consequent growing to the unstable phases of accretion which results finally in the formation of jets of matter from the cusp point, as the transition of orbiting matter through the regions $\Sigma_{u} \rightarrow \Sigma_{u}^{<} \rightarrow \Sigma_{u}^{\geq}$. Finally, the analysis of the dynamics in the region $\Sigma_{\varepsilon}^{+}$allows us to consider, from the starting condition, the extraction of energy and angular momentum of a black hole with the consequent release of particles (or matter jets), that could eventually induce a shift of the attractor from one class to another.

\subsection{Black holes}

The dynamical structure of $\Sigma_{\varepsilon}^{+}$in $\mathbf{B H}$ spacetimes is determined by the radii $r_{i} \in \mathscr{R}_{B H} \in \Sigma_{\varepsilon}^{+}$, as shown in Table 1 . The properties of circular orbits outside the ergoregion of blackhole geometries are sketched in Fig. 1, and they have been analyzed in detail in [19]. In the ergoregion, BH-sources are characterized by a unique family of corotating orbits with $L=L_{-}$. We summarize the results obtained from the analysis of $\Sigma$ by discussing the three classes of $\mathbf{B H}$ geometries: BHI : $a \in\left[0, a_{1}\right]$, BHII = BHIIa $\cup$ BHIIb : $\left.a \in] a_{1}, a_{2}\right]$, where the subclasses BHIIa $\left.\left.\equiv\right] a_{1}, a_{b}^{-}\right]$and BHIIb $\left.\equiv] a_{b}^{-}, a_{2}\right]$, and finally BHIII : $\left.\left.a \in\right] a_{2}, M\right]$ (see also Table 2).

\subsubsection{The class $\mathbf{B H I}: a \in\left[0, a_{1}\right]$}

The class BHI is bounded by the Schwarzschild static spacetime and the spacetime with spin $a=a_{1} \approx 0.707107 \mathrm{M}$. No circular motion can occur for $r<r_{\varepsilon}^{+}$, we have then $\Sigma_{\varepsilon}^{+}=\Sigma_{\varnothing}$ : the orbital amplitude of this region reaches its maximum in the limit geometry $a=a_{1}$, the static limit in this spacetime coincides with the photon orbit (see Fig. 1 and the discussion in Appendix C). There is no solution for Eq. (9), because the effective potential is always increasing $\left(V^{\prime}>0\right)$ in this region. A particle penetrating the ergoregion with $\dot{\theta}=0$ and $\dot{r}<0$ must move along the radial direction with a twisting along $\phi$. There is a belt, with boundary at $r_{\gamma}^{-}$, surrounding the static limit (in $r>r_{\varepsilon}^{+}$) where no inner edge for a thin disk ( $\mathrm{P}-\mathrm{W}$ point of accretion) and no cross point for the origin of matter jets can exist in the (outer) neighboring regions of the static limit. This forbidden region, however, decreases in extension as the spin-mass ratio approaches the upper limit of this class; its maximum value is in the limiting static geometry where its length is equal to the mass $M$ of the attractor. For spin values close to $a=a_{1}$ the forbidden belt decreases in length, until the launching point of the matter jet approaches the static limit at $r \gtrsim r_{\varepsilon}^{+}$.

\subsubsection{The class BHII : $\left.a \in] a_{1}, a_{2}\right]$}

The dynamical structure of the ergoregion in the case of BHII geometries is shown in Table 2 . The instability region con- 
tains the orbit $r_{b}^{-}$; it is therefore convenient to consider separately the subclass BHIIa : $\left.a \in] a_{1}, a_{b}^{-}\right]$and BHIIb : $a \in$ ]$\left.a_{b}^{-}, a_{2}\right]$. For a spacetime with $a_{b}^{-}=0.828427 M$ we have $r_{b}^{-}=r_{\varepsilon}^{+}$.

For BHIIa geometries with $a \neq a_{b}^{-}$we have $\Sigma_{u}\left(L_{-}\right)=$ $\Sigma_{\bar{u}}^{\geq}\left(L_{-}\right)$, which is a region with unstable unbounded $\left(E_{-}>\right.$ 1 ) orbits whose energy increases as the orbit approaches the photon orbit at $r_{\gamma}^{-}$. These orbits are unstable and under a perturbation they can eventually escape with $\dot{r}>0$ and $\dot{\phi}>0$, crossing the ergoregion at a certain finite time $\bar{\tau}: r(\bar{\tau})=r_{\varepsilon}^{+}$ and $\dot{r}(\bar{\tau})>0$ and $\dot{\phi}(\bar{\tau})>0$. The particle might run away to infinity, because the energy to decay into a lower circular orbit is higher than the energy required for spiraling outward to an exterior orbit. This could give rise to the ejection of positive energy particles outside the static limit. If we consider a thick accretion disk model, we could say that in the regions adjacent to the static limit or $] r_{\varepsilon}^{+}, r_{b}^{-}$] there can be only open configurations with a cross point in that region (or even in the static limit; see Appendix C), where there is a (critical) minimum pressure value, with the consequent launch of funnels of matter along the axis of symmetry. In fact, the cross point of an open configuration, i.e. the origin point of (corotating) matter jets, can occur in the ergoregion of BHIIa geometries in $\Sigma_{\bar{u}}^{\geq}\left(L_{-}\right) \in \Sigma_{\varepsilon}^{+}$, where the matter, twisting with the source (i.e. with initial angular momentum $L=L_{-}$), will be aligned with the rotation axis, while a part of the trapped particles in the ergoregion can be expelled to outside, without falling into the singularity. As shown in Table 2, the orbital extension $\mu_{u}^{\geq}$is less than $\mu_{\bar{u}}^{\geq}=0.26 M$ and the horizon is then covered by the orbit of the photon at $r_{\gamma}^{-}$. However, the static limit crosses the smaller region of instability as the attractor spin approaches the boundary of the class BHIIa, where close to the static limit, as $a=a_{b}^{-}$, there are accretion points of closed surfaces according to the $\mathrm{P}-\mathrm{W}$ accretion mechanism.

In BHIIb spacetimes, the region $\Sigma_{u}^{<} \subset \Sigma_{\varepsilon}^{+}$locates the instability points of the toroidal thick configuration of corotating matter in accretion. The hydrostatic pressure on the cusps is minimum, and these points can be even in the static limit (see Appendix C). No toroidal equilibrium disk can be inside the ergoregion due to the gravitational and hydrostatic instability in $\Sigma_{u}^{<}$. The point of accretion can also be very close to the static limit. Only open funnels are possible from a point in $\Sigma_{\bar{u}}^{\geq}$, where the launch of a jet is possible. The length of $\Sigma_{\bar{u}}^{\geq}$decreases, as the rotation of the attractor increases, approaching the horizon and creating therefore a gorge whose extension decreases from the maximum of $\mu_{u}^{\geq} \approx 0.12 M$ (see Table 2 ). In the proximity of the static limit $\left(r \gtrsim r_{\varepsilon}^{+}\right)$in BHIIb spacetimes, closed configurations in equilibrium are possible as $a \lesssim a_{2}$. The situation becomes critical as the attractor spin increases up to the upper limit of the BHIIb class with $a=a_{2}$. Then the closed configurations in equilibrium approach their center of maximum hydrostatic pressure, until the center coincides with the static
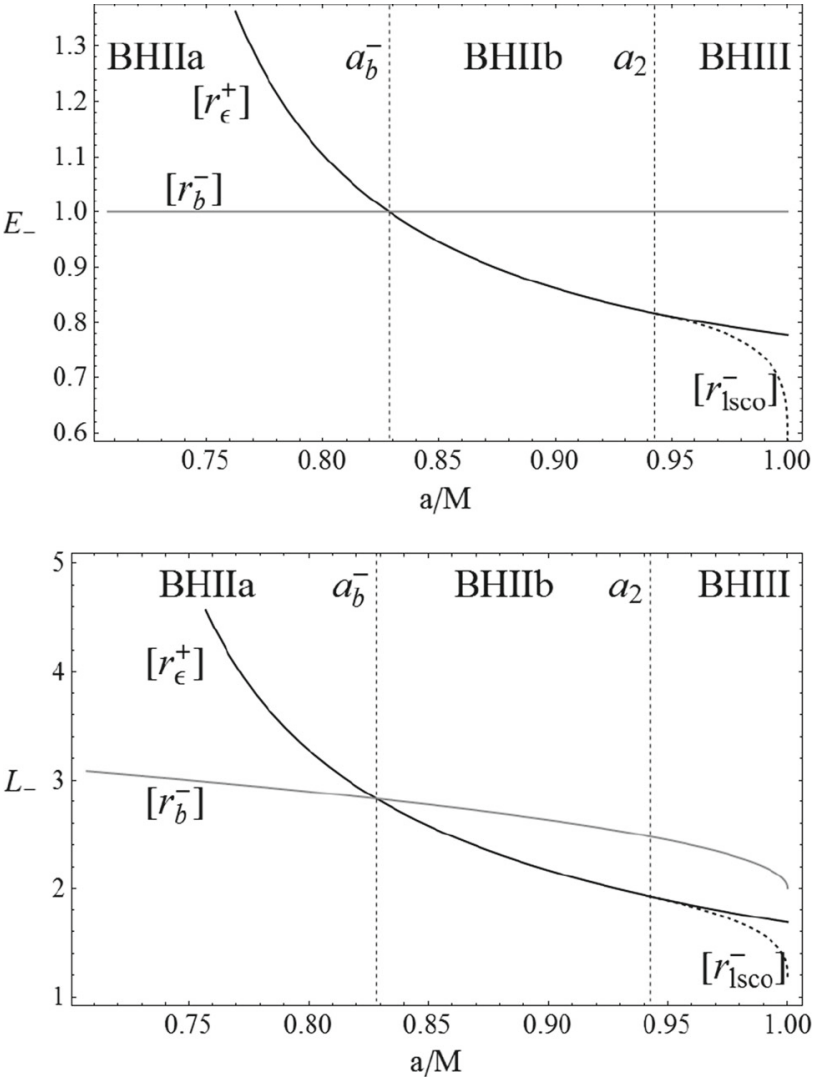

Fig. 2 Black-hole case: energies $E_{-}$(upper panel) and the angular momentum $L_{-}$(bottom panel) as functions of the spin-mass ratio $a / M$ of the black hole. The energies are in units of particle mass $\mu$ and the angular momentum in units of $M \mu$. The spins $a_{b}^{-}=0.828427 M, a_{2} \equiv$ $2 \sqrt{2} / 3 M$ are denoted by dashed lines. The curves are the energies and the angular momentum, respectively, for the specific orbits represented in square brackets next to the curves. $r_{\varepsilon}^{+}$is the static limit (black curve), $r_{b}^{-}$is the marginally bounded orbit (gray curve), $r_{l s c o}^{-}$is the last stable circular orbit (dashed curve)

limit in the spacetime with $a=a_{2}$. Then the inner edge of the disk would be within the region $\Sigma_{\bar{u}}^{\geq}$. The energy $E_{-}>0$ and orbital angular momentum $L_{-}>0$ increase, approaching the limiting value at $r_{\gamma}^{-}$(see also Fig. 3). On the other hand, at a fixed orbit $r$, the energy decreases as the attractor spin increases. As shown in Fig. 2, the minimum energy extractable during an orbital decay in BHIIa geometries is $E=\mu$ for the orbit $r_{b}^{-}$. The maximum extractable energy from an initial decay by a particle in $\Sigma_{u}^{\geq}$does not exceed the value $E=1.6 \mu$ corresponding to a particle that decays from an orbit very close to the static limit. In BHIIb spacetimes, the minimum extractable energy is not less than $E=0.8 \mu$ as shown in Fig. 2.

\subsubsection{The class BHIII : $\left.a \in] a_{2}, M\right]$}

In BHIII spacetimes, there can be stable orbits in the ergoregion $\Sigma_{\varepsilon}^{+}$(see Fig. 1; Table 2). As we have $r_{\varepsilon}^{+} \in \Sigma_{s}$, the static limit can correspond to a stable orbit, according to the 


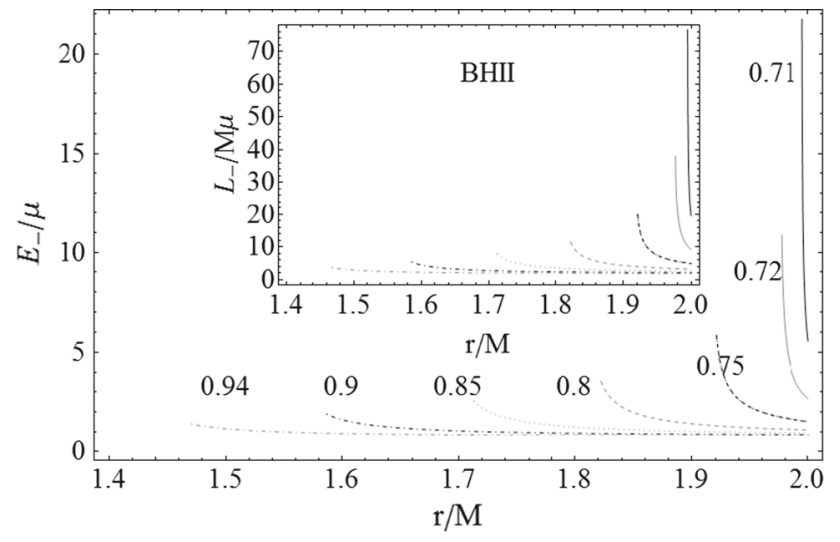

Fig. 3 The energy $E_{-}$and the corresponding angular momentum $L_{-}$ (inside panel) as functions of $r / M$ and for different values of the spinto-mass ratio of BHII sources

discussion of Sect. 2.1 (see also Appendix C). There may exist a stable thin ring of dust orbiting very close around $r_{\varepsilon}^{+}$ $\left(r=r_{\varepsilon}^{+} \pm \varepsilon\right.$ with $\left.\varepsilon \gtrsim 0\right)$. The inner edge and the center (maximum of the hydrostatic pressure) of the thin disk can be in $\Sigma_{s} \subset \Sigma_{\varepsilon}^{+}$. The length $\mu_{s}$ of this region increases as $a \lesssim M$. A thick corotating accretion disk in BHIII spacetimes can be entirely inside $\Sigma_{\varepsilon}^{+}$, dynamically including the ring formation in equilibrium up to the accretion and the jet launch. Thus the point of launch of the jet, according to the $\mathrm{P}-\mathrm{W}$ mechanism, can be located in a region very close to the horizon, as the attractor spin increases. A slightly change in the attractor spin would produce a transition from a section to another inside the ergoregion, causing a change in the stability properties of the orbital matter. However, the maximum length $\mu_{s}$ of the region of stability is $\mu_{s}=M$; therefore, the elongation of a thin disk entirely contained in $\Sigma_{\varepsilon}^{+}$cannot be greater than $M$.

As the largest section for a fixed attractor is $\Sigma_{u}^{<}$, one could say that closed stable configurations are favored in BHIII spacetimes, jets in BHIIa sources, and the accretion disks in BHIIb and BHIII attractors. The behavior of the energy and angular momentum in these spacetimes is shown in Fig. 2. The maximum energy can be extracted from a particle that decays from an orbit close to the static limit and slightly greater than $E \approx 0.8 \mu$, while the angular momentum does not exceed the value $L \approx 2 M \mu$.

\subsection{Naked singularities}

We distinguish three classes of naked singularity sources: NSI : $\left.a \in] 1, a_{3}\right]$, split into NSIa: $\left.\left.a \in\right] 1, a_{\mu}\right]$ and NSIb: $\left.a \in] a_{\mu}, a_{3}\right]$, the class NSII : $\left.\left.a \in\right] a_{3}, a_{4}\right]$, and finally the class NSIII : $\left.a \in] a_{4}, \infty\right]$, split into NSIIIa : $\left.\left.a \in\right] a_{4}, a_{b}^{N S}\right]$ and NSIIIb : $a \in] a_{b}^{N S}, \infty[$ (see also Table 1). A characterization of the orbital energy in $r>r_{\varepsilon}^{+}$can be found with some details in [19]. In contrast with $\mathbf{B H}$, in $\mathbf{N S}$ spacetimes the singularity is always covered by the region $\Sigma_{\bar{u}}^{\geq}\left(L_{-}\right)$, with unstable and unbounded circular orbits.

\subsubsection{The class NSI : $\left.a \in] 1, a_{3}\right]$}

Naked singularities of the NSI class present a rather rich and articulated dynamical structure of the ergoregion, as shown in Table 3. As they limit with the extreme-BH case at $a=M$, NSI spacetimes could be involved in the hypothetical transitions between the $\mathbf{B H}$ and NS classes. In contrast with the $\mathbf{B H}$ spacetimes, these geometries are characterized by two types of circular orbits: the corotating orbits with momentum $L=L_{-}$, which extend to the regions $r>r_{\varepsilon}^{+}$, and the counterrotating ones with $L=-L_{-}$, confined in a bounded orbital region $\Sigma\left(-L_{-}\right) \subset \Sigma_{\varepsilon}^{+}$with boundaries at $r=\hat{r}_{ \pm}$, as defined in Eq. (A.7), corresponding to

Table 3 Dynamical structure of the ergoregion $\Sigma_{\varepsilon}^{+}$and length $\mu$ of its sections in naked singularity geometries: the label ()$_{\varnothing}$ refers to the regions where circular orbits are not allowed, ()$_{s}$ to regions with stable circular orbits, ()$_{u}^{<}$to regions with unstable orbits with energy $|E|<1$ (in units of the mass particle), and finally ()$_{\vec{u}}^{\geq}$is for the regions where unstable circular orbits exist with $|E|>1$. The corresponding lengths are denoted by $\mu_{()}$. The boundaries of the ranges for the length $\mu_{()}$correspond to values of the boundary geometries of each spacetime class. The regions $\Sigma$, and the corresponding lengths $\not \mu$, refer to orbits with negative energy. The regions are ordered according to the decreasing orbital distance from the source, that is, from the more distant from the source to the closest. The lengths are in units of mass $M$. For details, see Fig. 4. The angular momentum characterizing the possible orbits are in round brackets. There are no regions with both corotating $\left(L_{-}\right)$ and counterrotating $\left(-L_{-}\right)$angular momenta. The two kinds of orbits are confined in disjoint regions

Naked singularity geometries:

NSIa: $\Sigma_{\varepsilon}^{+}=\Sigma_{u}^{-}\left(L_{-}\right) \cup \Sigma_{s}\left(-L_{-}\right) \cup Z_{s}\left(-L_{-}\right) \cup Z_{u}^{<}\left(-L_{-}\right) \cup$ $\Sigma_{u}^{<}\left(-L_{-}\right) \cup \Sigma_{u}^{<}\left(L_{-}\right) \cup \Sigma_{u}^{\geq}\left(L_{-}\right)$

Length of the sections: $\mu_{u}^{<}\left(L_{-}\right) \in[1,1.00873], \mu_{s}\left(-L_{-}\right)$ $\in[0,0.324601], \mu_{s}\left(-L_{-}\right) \in[0,0]$,

$\mu / u\left(-L_{-}\right) \in[0.618034,0], \mu_{u}\left(-L_{-}\right) \in[0.0863683,0.298875]$, $\mu_{u}^{<}\left(L_{-}\right) \in[0.124025,0.16957]$,

$\mu_{u}^{\geq}\left(L_{-}\right) \in[0.171573,0.198221]$.

NSIb: $\Sigma_{\varepsilon}^{+}=\Sigma_{u}^{-}\left(L_{-}\right) \cup \Sigma_{u}^{<}\left(-L_{-}\right) \cup \Sigma_{S}\left(-L_{-}\right) \cup \Sigma_{u}^{<}\left(L_{-}\right)$ $\cup \Sigma_{u}^{\geq}\left(L_{-}\right)$

Length of the sections: $\mu_{u}^{<}\left(L_{-}\right) \in[1.00873,1.25]$, $\mu_{s}\left(-L_{-}\right) \in[0.324601,0]$,

$\mu_{u}\left(-L_{-}\right) \in[0.298875,0], \mu_{u}^{<}\left(L_{-}\right) \in[0.16957,0.483478]$, $\mu_{u}^{\geq}\left(L_{-}\right) \in[0.198221,0.266522]$

NSII: $\Sigma_{\varepsilon}^{+}=\Sigma_{s}\left(L_{-}\right) \cup \Sigma_{u}^{<}\left(L_{-}\right) \cup \Sigma_{u}^{\geq}\left(L_{-}\right)$

Length of the sections: $\mu_{s} \in[1.25,0], \mu_{u}^{<} \in[0.483478,1.08485]$, $\mu_{u}^{\geq} \in[0.266522,0.915154]$

NSIIIa: $\Sigma_{\varepsilon}^{+}=\Sigma_{u}^{<}\left(L_{-}\right) \cup \Sigma_{u}^{\geq}\left(L_{-}\right)$

Length of the sections: $\mu_{u}^{<} \in[1.08485,0], \mu_{u}^{\geq} \in[0.915154,2]$

NSIIIb: $\Sigma_{\varepsilon}^{+}=\Sigma_{u}^{\geq}\left(L_{-}\right)$

Length of the sections: $\mu_{\bar{u}}^{\geq}=2$ 

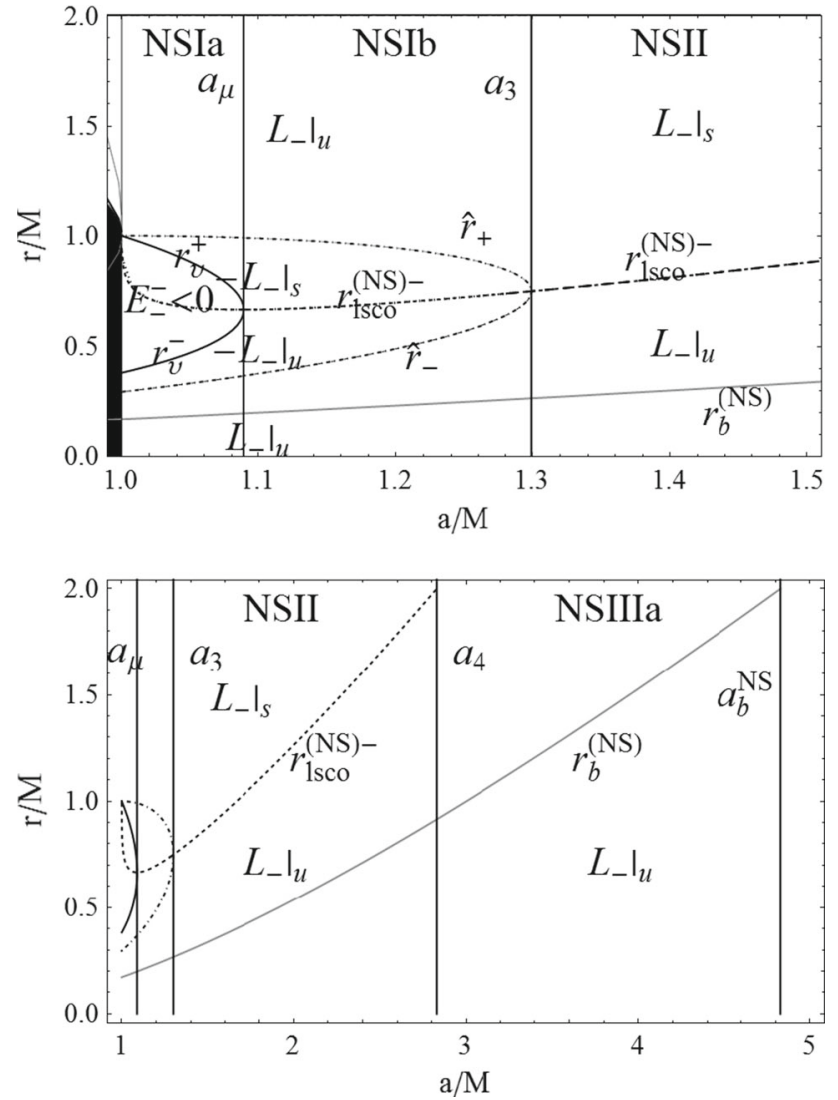

Fig. 4 Arrangement of the radii $r_{i} \in \mathscr{R}_{N S}$ determining the properties of circular orbits around a rotating naked singularity (NS) in the region $\Sigma_{\varepsilon}^{+}$. The orbital stability in each region is analyzed and explicitly shown. The angular momentum is quoted with its stability property as $\left.\right|_{s}$ for stable and $\left.\right|_{u}$ for unstable orbits. $r_{b}^{(N S)}$ corresponds to the marginally bounded orbits, $r_{l s c o}^{(N S)-}$ to the marginally stable orbits. On $\hat{r}_{ \pm}$, the orbits have zero angular momentum $(L=0)$. The radii $r_{v}^{ \pm}$correspond to counterrotating $\left(L=-L_{-}\right)$orbits with zero energy $(E=0)$. The region $] r_{v}^{-}, r_{v}^{+}$[ contains only counterrotating orbits with $E<0$

circular orbits with zero angular momentum $L=0$. The orbital region $\Sigma\left(-L_{-}\right)$is split by the radius $r_{l s c o}^{(N S)-}$ so that $\Sigma\left(-L_{-}\right)=\Sigma_{u}^{<}\left(-L_{-}\right) \cup \Sigma_{s}\left(-L_{-}\right)$. These regions do not intersect each other, i.e., $\Sigma\left(-L_{-}\right) \cap \Sigma\left(L_{-}\right)=\emptyset$, in contrast to the region $r>r_{\varepsilon}^{+}$, where it is possible to have, in the same orbital region, corotating and counterrotating matter. The regions $\Sigma\left(-L_{-}\right)$and $\Sigma\left(L_{-}\right)$are completely disjointed in $\Sigma_{\varepsilon}^{+}$, although the presence of circular orbits with $L=0$ at $\hat{r}_{ \pm}$suggests a continuous transition between the two regions. For orbits with $L=0$ at $r=\hat{r}_{ \pm}$, the particle energy is positive and increases with the source spin, with the condition $E\left(\hat{r}_{-}\right)>E\left(\hat{r}_{+}\right)$(see Figs. 5 and 6). The existence of counterrotating orbits can be seen as a "repulsive gravity" effect that has been detected also in other naked singularity spacetimes $[25,26,28]$, creating therefore an "antigravity" sphere bounded by orbits with zero angular momentum. The stabil- ity of these orbits would suggest the presence of a belt of counterrotating material covering the singularity.

In $\Sigma\left(-L_{-}\right)$, there is a class of naked singularities NSIa $\equiv$ $\left.a \in] M, a_{\mu}\right]$ where circular counterrotating orbits are possible with negative energy, $\mathbb{Z}=\Psi_{s}\left(-L_{-}\right) \cup Z_{u}^{<}\left(-L_{-}\right)$. The energy of the particles in these orbits is always less than $\mu$ in magnitude. The region $₹$ is bounded by the orbits $r_{v}^{ \pm}$, zero energy orbits (see Fig. 4). Counterrotating particles are confined inside the closed region $\Sigma_{s}\left(-L_{-}\right) \cup \Sigma_{u}^{<}\left(-L_{-}\right)$in NSIa $\cup$ NSIIb with a section of negative energy particles $Z_{s}\left(-L_{-}\right) \cup Z_{u}^{<}\left(-L_{-}\right)$in NSIa. The symbol $\Sigma$ is used in relation with the regions of negative energy orbits and, unless otherwise specified, $\Sigma$ will be considered for positive energy orbits, while the labels $(\geq)$ and $(<)$ are to be understood, in the case of negative energies, as related to the energy magnitude.

The existence of stable and unstable circular orbits with $L<0$ and $E<0$, although located in an orbital region far from the source, can be important for the phenomena of accretion from the equatorial plane, because it would imply dropping "test" material into the singularity with a negative contribution to the total energy and momentum. The region of stable orbits is disconnected in the sense discussed previously in [19,25-28], i.e., if we imagine a hypothetical accretion disk made of test particles only, the disconnected stability regions form a ringlike configuration around the central object [25-27].

The existence of the region $\Sigma$ is an intrinsic characteristic of NS-sources that has also been highlighted for other axisymmetric exact solutions of the Einstein equations, in particular, for the electrovacuum spacetime described by the Reissner-Nordström (RN) solution. ${ }^{4}$ In this spacetime, the radii where $E=0$ define the effective ergoregion of the RN solution [43] or ] $r_{+}^{\mathrm{RN}}, r_{\text {eff }}^{\varepsilon}\left[\right.$, where $r_{+}^{\mathrm{RN}} \equiv M+\sqrt{M^{2}-Q^{2}}$ is the outer horizon in the RN geometry and $r_{\text {eff }}^{\varepsilon} \equiv M+$ $\sqrt{M^{2}-Q^{2}\left(1-q^{2} / \mu^{2}\right)}$, where $Q$ is the source charge and $q$ is the test particle charge. The existence of such a region is due to the attractive electromagnetic interaction between the two charges $(Q q<0)$, resulting in negative energy states for test particles [26,27]. However, since the RN solution is static, there is no ergoregion in this spacetime.

The definition of an effective ergoregion is introduced in the description of the energy extraction phenomena of black holes, and can be extended also to the case of naked singulari-

\footnotetext{
${ }^{4}$ In general, even if the global structure of the Kerr spacetime is $\theta$ dependent, in the equatorial plane these two axisymmetric solutions have some remarkable similar geometrical features; for instance, in the expressions for the outer and inner horizons $r_{ \pm}$, where the horizons of the one solution can be fit into the outer by replacing the spin parameter $a / M$ of the Kerr spacetime with the electric charge parameter $Q / M$ of the RN solution. Finally, in the equatorial plane the conformal diagram for the maximally extended Kerr (BH and NS) spacetimes is identical to that of the RN solution [41].
} 

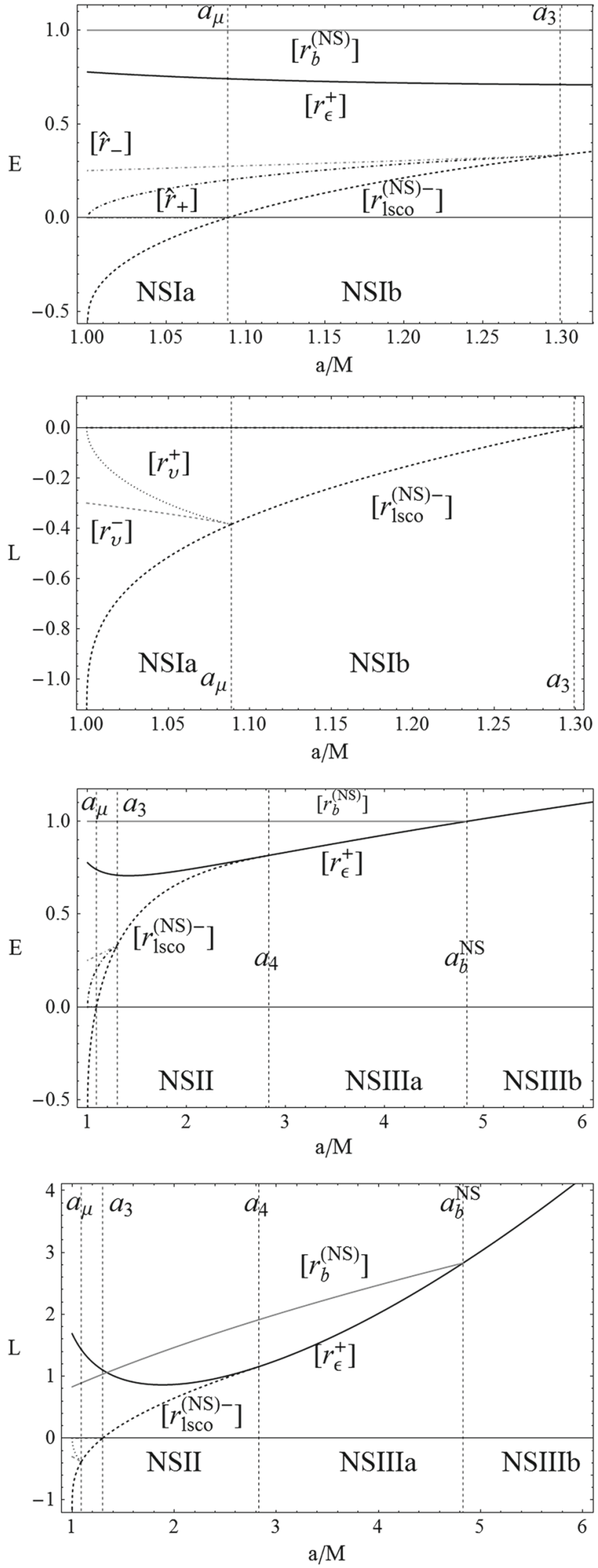

4 Fig. 5 The naked singularity case: the energies $E$ and angular momenta $L$ as functions of the spin-mass ratio $a / M$ of the naked singularity. The energies and the angular momenta are in units of the particle mass $\mu$. The spins $a_{3} / M \equiv 3 \sqrt{3} / 4, a_{\mu} / M \equiv 4 \sqrt{2 / 3} / 3$, $a_{b}^{N S} / M \equiv 2(1+\sqrt{2}) \approx 4.82843$, and $a_{4} / M=2 \sqrt{2}$ are denoted by dashed lines. The curves represent the energies and the angular momenta, respectively, for specific orbits marked in square brackets next to the curves. $r_{\varepsilon}^{+}$is the static limit, $r_{b}^{(N S)}$ is the marginally bounded orbit, $r_{l s c o}^{(N S)-}$ is the last stable circular orbit. At $\hat{r}_{ \pm}$, we have $L\left(\hat{r}_{ \pm}\right)=0$, and at $r_{v}^{ \pm}$(effective ergosurfaces), we have $E\left(r_{v}^{ \pm}\right)=0$

ties. Therefore, one can define also for the NSIa singularities the radii $r_{v}^{ \pm}(a)$, where $E=0$, as the outer effective ergosurface $r_{v}^{+}$and an inner effective ergosurface $r_{v}^{-}$. Then the region ] $r_{v}^{-}, r_{v}^{+}$[ would correspond to an effective ergoregion, where the spin-orbit coupling terms in the Kerr source play the role of the electrodynamic interaction between the test charge $q$ and the intrinsic charge of the source in the definition of the effective ergoregion in the RN geometry. However, in the Kerr spacetimes, the radii $r_{v}^{ \pm}(a)$ are independent of the orbital angular momentum.

\subsubsection{The class NSII : $\left.a \in] a_{3}, a_{4}\right]$}

The dynamical structure of $\Sigma_{\varepsilon}^{+}$in the NSII geometries is shown in Table 3 and in Figs. 1 and 4. There are only corotating orbits with $L=L_{-}>0$, which can be stable or unstable, bounded and unbounded, so that the structure of $\Sigma_{\varepsilon}^{+}$is similar to the case of BHIII as given in Table 2. In the NSII class, the region $\Sigma_{\bar{u}}^{\geq}\left(L_{-}\right)$extends up to the singularity and there is no region $\Sigma_{\varnothing}$. A further difference with BHIII spacetimes is that the length $\mu_{s}$ of $\Sigma_{s}$ in NSII decreases with the attractor spin. This can be interpreted as follows: while higher spins in the $\mathbf{B H}$ geometries favor the orbital stability, an increase of the attractor spin in the NS spacetimes acts in the opposite direction, favoring the instability of the (unbounded) orbits. A pressure supported accretion disk can then be entirely contained in $\Sigma_{\varepsilon}^{+}$, and eventually evolve toward the instability, moving the inner edge of the disk toward the region $\Sigma_{u}^{<}$, and eventually toward $\Sigma_{u}^{\geq}$, giving raise to funnels of materials launched outward from the minimum point of the hydrostatic pressure. The length of the region where the center of the closed configuration can be located does not exceed $\mu_{s}=1.25 \mathrm{M}$, while it decreases to zero as the singularity spin reaches the upper limit $a_{4}$ of this class of sources. Moreover, in the wider region, where a fixed attractor is in $\Sigma_{u}^{<}$with a minimum length of $\mu_{u}^{<} \approx 0.48 M$ and a maximum of $\mu_{u}^{<} \approx 1.085 M$, there are toroidal surfaces close around the maximum of the pressure and points of instability in $\Sigma_{u}^{<}$from which there could be an overflow 

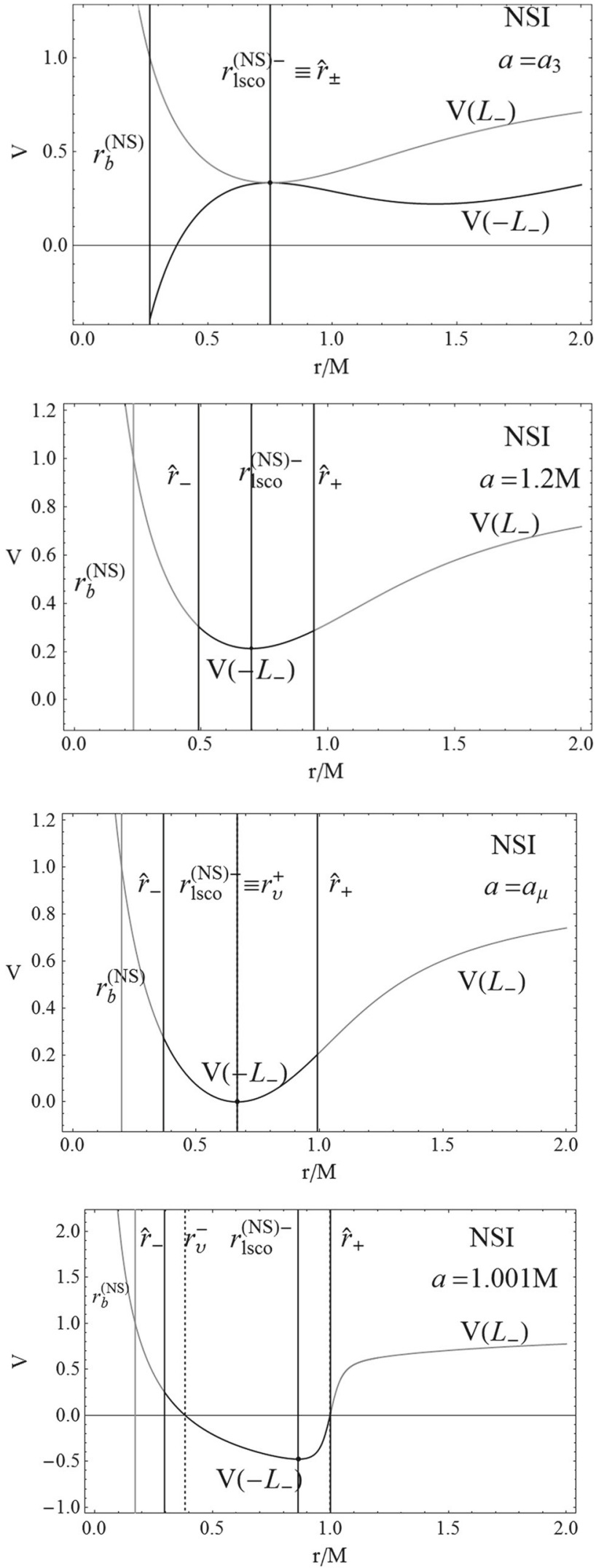

4 Fig. 6 NSI sources with $\left.a \in] M, a_{3}\right]$. The energy $E_{-}^{-} \equiv V\left(-L_{-}\right)$ (black curves) and $E_{-} \equiv V\left(L_{-}\right)$(gray curves), in units of the particle mass $\mu$, of the circular orbits for different spacetime spins. The last stable circular orbit $r_{l s c o}^{(N S)-}$ il also plotted and the minimum of the energy is marked with a point. At $a=a_{3}$ we have $\hat{r}_{ \pm}=r_{\text {lsco }}^{(N S)-}$. The potential $V\left(-L_{-}\right)$is plotted for the entire range $\left.\left.r \in\right] 0, r_{\varepsilon}^{+}\right]$, but the only counterrotating orbit is located at the boundary point $\hat{r}_{ \pm}=r_{l s c o}^{(N S)-}$. For spacetimes with $a \in] M, a_{\mu}$ [ the energy of the circular orbit is negative, and for a source with $a=a_{\mu} \equiv(4 \sqrt{2 / 3}) / 3 M$ the energy vanishes, $E_{-}^{-}\left(r_{l s c o}^{(N S)-}, a_{\mu}\right)=0$

of matter. As shown in Fig. 5, the orbit energies and the angular momenta increase with the spin of the source, and they can reach a minimum in the static limit (see Appendix C). In order to set test particles in circular orbits in these spacetimes we should provide a specific energy that increases with the attractor spin.

\subsubsection{The class NSIII : $a \in] a_{4},+\infty[$}

In NSIII geometries, as in the BHII class of attractors, the marginally bounded orbit $r_{b}^{(N S)}$ crosses the static limit as the spacetime spin is $a=a_{b}^{N S} \equiv 2(1+\sqrt{2}) M \in \mathbf{N S I I I}$ (see Table 3 and Fig. 4). It is therefore convenient to introduce a further decomposition of this class into two subclasses, namely NSIIIa where $\left.a \in] a_{4}, a_{b}^{N S}\right]$ and NSIIIb where $a>a_{b}^{N S}$. In all NSIII spacetimes, the stable orbits are suppressed for NSIIIa attractors, but bounded unstable orbits are still possible. In the NSIIIb spacetimes the ergoregion can be filled only by unbounded orbits up to the singularity, and the dynamical structure of the ergoregion reduces to $\Sigma_{\varepsilon}^{+}=\Sigma_{\bar{u}}^{\geq}$. In the $\Sigma_{u}$ regions, the particle orbits are unstable and, correspondingly, extended pressure supporting toroidal matter orbiting around these attractors, have instability points located in $\Sigma_{u}^{<}$, where an overflow of matter is possible from a closed configuration. Moreover, there can also be jet launches with funnels of materials in $\Sigma_{\bar{u}}^{\geq}$, which characterizes in particular the whole ergoregion of the NSIIIb spacetimes.

On the other hand, only in the NSIIIa geometries the disk center can be located in the region $r>r_{\varepsilon}^{+}$close to the static limit. In this respect, the dynamical structure of $\Sigma_{\varepsilon}^{+}$in NSIIIa and NSIIIb spacetimes is similar to the BHIIb and BHIIa classes, respectively.

We can conclude that naked singularities with sufficiently high spins, strong naked singularities of the NSIIIa class, and black holes with sufficiently small spin within the BHIIb class do not allow for any stable orbiting matter in the ergoregion. The orbital energy and angular momentum of the circular orbits in NSIII spacetimes increase with the singularity spin, as shown in Fig. 5. Bounded orbits are not allowed in very strong naked singularities of the NSIIIb 
class, but they can exist in weak black holes of the BHIIa class with lower spin (see Fig. 1). An increase of the attractor spin in weak black-hole geometries, or a decrease in strong and very strong naked singularities, affects positively the process of the formation of a circular orbiting configuration.

\section{Conclusions}

In this work, we have performed a complete analysis of the properties of circular motion inside the ergoregion of a Kerr spacetime. From the physical point of view, the ergosurface is a particularly interesting surface, because it represents the limit at which an observer can stay at rest. In the ergoregion, an observer is forced to move due to the rotation of the gravitational source.

The dynamics inside the ergoregion is relevant in astrophysics for the possible observational effects, as the matter can eventually be captured by the accretion, increasing or removing part of its energy and angular momentum, prompting a shift of its spin, and inducing an unstable phase where the intrinsic spin changes (spin-down and spin-up processes with the consequent change in the causal structure). A discussion of the ergoregion stability can be found in [49,50]. For further consideration of a possible destruction of the horizon and naked singularity formation, see, for example, [6,51-55]. It is therefore possible that, during the evolutionary phases of the rotating object, the interaction with orbiting matter could lead to evolutionary stages of spin adjustment, in particular, for example, in the proximity of the extreme Kerr solution (with $a \lesssim M$ ) where the speculated spin-down case of the BH can occur, preventing the formation of a naked singularity with $a \gtrsim M$ (see also [5,7,10,44,56-61,65]). On the other hand, the accreting matter can even get out, giving rise, for example, to jets of matter or radiation [1]. However, it is important to emphasize that different processes can lead to jet emission from black-hole accretion disks; in general, it is widely believed that a crucial role is played by the electromagnetic field, giving rise to magnetically driven jets. Another possibility is the extraction of energy from a rotating black hole through the Blandford-Znajek mechanism. See, for example, [66-75] for general discussions of the role of magnetic fields in the formation of jets. A possible generalization of the present work to include this mechanism would imply a thorough study of the effects of the electromagnetic field on the particle acceleration in the spacetime of a rotating black hole. Several aspects of this problem have been addressed in the literature, for example, in [76-79] where it was investigated how the magnetic field affects the motion of test particles in the vicinity of a black hole. Further discussions of the role of the electromagnetic fields in black-hole physical processes can be found for example in $[80,81]$.
In our analysis, we limit ourselves to the study of circular orbits located in the equatorial plane of the Kerr spacetime. On the equatorial plane, the static limit does not depend on the source spin, but for any Kerr spacetime it is $r_{\varepsilon}^{+}=2 M$ and it coincides with the event horizon of the Schwarzschild spacetime. This is a simple setting that allows for an immediate comparison with the limiting case $a=0$. Furthermore, matter configurations in accretion are typically axially symmetric and many of the geometrical and dynamical characteristics of the disk are determined by the properties of the configuration in the accretion equatorial section.

Our approach consists in rewriting the geodesic equations in such a form that the motion along circular orbits is governed by one single ordinary differential equation whose properties are completely determined by an effective potential. The conditions imposed on the effective potential for the existence of circular motion allow us to derive explicit expressions for the energy and angular momentum of the test particle. The behavior of these physical quantities determines the main properties of the circular orbits in terms of the radial distance which, in this case, coincides with the radius of the orbit, and the intrinsic angular momentum of the gravitational source. We performed a very detailed investigation of all the spatial regions inside the ergoregion where circular motion is allowed. In addition, we investigate the stability properties of all the existing circular orbits.

The distribution of circular orbits inside the ergoregion turns out to depend very strongly on the rotation parameter $a$ of the source, and this makes it necessary to split the analysis into two parts: black holes and naked singularities. In addition, the behavior of the effective potential in the ergoregion in terms of the rotational parameter suggests an additional split by means of which black holes become classified in four classes, namely, BHI, BHIIa, BHIIb, and BHIII, whereas naked singularities are split into five classes, namely, NSIa, NSIb, NSII, NSIIIa, and NSIIIb. We then investigate in detail for each class the behavior of the energy and angular momentum of the test particle, as well as the properties of the effective potential. In this manner, the analysis of circular motion allows us to derive physical information as regards entire sets of black holes and naked singularities.

Circular motion is possible inside the ergoregion in black holes and naked singularities as well. However, there are fundamental differences if we consider the stability properties. In the case of black holes, only the set BHIII can support a spatial region with particles moving along circular corotating stable orbits. The BHIII class includes all the black holes whose rotation parameter is contained within the interval $a / M \in] 2 \sqrt{2} / 3$, 1], i.e., rapidly rotating black holes including the extreme black hole. The spatial region with stable particles extends from the radius of the ergoregion $\left(r_{\varepsilon}^{+}=2 M\right)$ to the radius of the last stable circular orbit, so that the maximum radial extension of this region is $M$ for an extreme black 
hole, and the minimum extension is zero for a black hole with $a=2 \sqrt{2} / 3 M$. The last case corresponds to particles moving in the last stable circular orbit. If we imagine a hypothetical accretion disk made of test particles only, then we conclude that black holes can support inside the ergoregion only one corotating disk with a maximum extension of $M$. Introducing the concept of dynamical structure of the ergoregion, we have discussed also the properties of toroidal extended configurations (pressure supported hydrodynamic accretion disk models) in the regions that determine the dynamical structure. This structure is formed by a (uniquely defined) collection of disjoint regions of the orbit plane whose union recomposes the ergoregion. A change in the orbit, at fixed spin, can lead to a shift in the dynamical structure, while a change of the attractor spin can lead to a transition between the regions; moreover, a shift in the boundary spins is equivalent to a transition between classes and sometimes the regions of the structure. The stability analysis, with respect to a change in the spin of the source, has led to the identification of special $\mathbf{B H}$ sources (with $a=a_{b}^{-} / M \approx 0.828427$ and $a=a_{2} / M \approx 0.942809$ ), where a possible change in the value of the spin, in the margins between the different regions of the dynamical structure, affects appreciably the properties of the toroidal structures, indicating that this kind of structures is more sensitive to a change of the source.

The case of naked singularities is more complex. In fact, one of the interesting features is that inside the ergoregion there can exist particles moving along counterrotating stable orbits. As a consequence, the location and structure of the regions with stable particles is much more complex than in the case of black holes. In particular, it was shown that there can exist several stability regions, separated by instability regions. This implies a discontinuous structure of the stability regions so that, if certain energy conditions are satisfied, an accretion disk made of test particles would show a ringlike structure. This makes naked singularities essentially different from black holes. The characteristics of the rings and their extensions depend on the explicit value of the rotation parameter. Finally, we found that there exists a maximum value of the spin for which no more stable configurations can exist, namely, $a=2 \sqrt{2} M \approx 2.828 M$ where the radius of the last stable circular orbit coincides with the radius of the ergoregion. Naked singularities with spins greater than this critical value do not support any stable disklike or ringlike configurations entirely contained in the ergoregion. In NSI spacetimes, there are both stable and unstable counterrotating orbits inside the ergoregion. This fact can be understood as the effect of repulsive gravity, but it is interesting to note that this phenomenon can occur only in sufficiently slowly rotating naked singularities with spin values close to the value of the extreme BH case. In NSII spacetimes, there can exist stable corotating orbits; this is the major difference with the $\mathbf{B H}$ case and it represents also the main dif- ference with the other NS sources. We have introduced the concepts of inner and outer effective ergosurfaces defined by the radii $r_{v}^{-}$and $r_{v}^{+}$, where $E=0$. The effective ergoregion (at $r \in] r_{v}^{-}, r_{v}^{+}$[) is defined for supercritical configurations with $a \in] M, 1.08866 M]$ in NSI spacetimes, where $E<0$. Our results show that the complex stability properties of circular orbits inside the ergoregion of naked singularities is due to the presence of effects that can be interpreted as generated by repulsive gravitational fields. The nature of this type of fields is not known. We expect to investigate this problem in future work by using certain invariant properties of repulsive gravity [62]. For the sake of completeness, we have also investigated all the properties of circular orbits in the limiting case of extreme black holes, classifying all the sources that allow for circular orbits at the radius of the static limit. In both cases, we used the available physical parameters to perform a detailed analysis confirming the rich structure of the gravitational sources described by the Kerr spacetime.

Acknowledgments This work was supported in part by DGAPAUNAM, Grant No. 113514, and Conacyt, Grant No. 166391. DP gratefully acknowledges financial support from Blanceflor BoncompagniLudovisi, née Bildt, and would like to thank the institutional support of the Faculty of Philosophy and Science of the Silesian University of Opava. DP thanks an anonymous colleague who has read in great detail the work, providing us with important suggestions to improve the presentation of the results.

Open Access This article is distributed under the terms of the Creative Commons Attribution 4.0 International License (http://creativecomm ons.org/licenses/by/4.0/), which permits unrestricted use, distribution, and reproduction in any medium, provided you give appropriate credit to the original author(s) and the source, provide a link to the Creative Commons license, and indicate if changes were made.

Funded by SCOAP ${ }^{3}$.

\section{Appendix A: Stability of circular orbits and notable radii}

The last circular orbits, $r_{\gamma} \in\left\{r_{\gamma}^{-}, r_{\gamma}^{+}\right\}$, are located at

$r_{\gamma}^{\mp} \equiv 2 M\left(1+\cos \left[\frac{2 \arccos \left(\mp \frac{a}{M}\right)}{3}\right]\right)$,

where $r_{\gamma}^{-} \in \Sigma_{\varepsilon}^{+}$in the $\mathbf{B H}$ geometries. The stability properties of the particle circular dynamics are regulated by the second radial derivative of the effective potential: the saddle points of the function $V$, given by the conditions $V^{\prime}=0$ and $V^{\prime \prime}=0$, define the radii of the last stable circular orbits $r_{l s c o} \in\left\{r_{l s c o}^{\mp}, r_{l s c o}^{(N S)}, r_{l s c o}^{(N S)-}\right\}$, where

$r_{l s c o}^{\mp} \equiv M\left(3+Z_{2} \mp \sqrt{\left(3-Z_{1}\right)\left(3+Z_{1}+2 Z_{2}\right)}\right)$,

$r_{l s c o}^{-}$for $\mathbf{B H}: L=L_{-}, \quad r_{l s c o}^{+}$for $\mathbf{B H} / \mathbf{N S}: L=-L_{+}$.

Here $r_{l s c o}^{-}$is for corotating with $L=L_{-}$orbits in a blackhole geometry and $r_{l s c o}^{+}$is for counterrotating orbits with 
$L=-L_{+}$in black-hole and naked singularity spacetimes, respectively, and

$$
\begin{aligned}
& r_{l s c o}^{(\mathrm{NS})-} \equiv M\left[3-Z_{2}+\sqrt{\left(3-Z_{1}\right)\left(3+Z_{1}-2 Z_{2}\right)}\right] \\
& \text { for } a / M>1.28112 \text { and } L=L_{-}, \\
& r_{l s c o}^{(\mathrm{NS})-} \equiv M\left[3-Z_{2}-\sqrt{\left(3-Z_{1}\right)\left(3+Z_{1}-2 Z_{2}\right)}\right] \\
& \text { for } a / M \in] 1,1.28112\left[\text { and } L=-L_{-},\right.
\end{aligned}
$$

where $a=1.28112 M \in \mathbf{N S I}^{5}$ and $Z_{2} \equiv \sqrt{3(a / M)^{2}+Z_{1}^{2}}$ where

$Z_{1} \equiv 1+\left[1-(a / M)^{2}\right]^{1 / 3}\left[(1+a / M)^{1 / 3}+(1-a / M)^{1 / 3}\right]$.

We have $\left(r_{l s c o}^{-}, r_{l s c o}^{(N S)-}\right) \in \Sigma_{\varepsilon}^{+}$.

The marginally bounded orbits, $r_{b} \in\left\{r_{b}^{ \pm}, r_{b}^{(N S)-}\right\}$, are located at

$r_{b}^{ \pm} \equiv 2 M \pm a+2 \sqrt{M} \sqrt{M \pm a}$,

$r_{b}^{-}$for $\mathbf{B H}: L=L_{-}, \quad r_{b}^{+}$for $\mathbf{B H} / \mathbf{N S}: L=-L_{+}$

for particles corotating with angular momentum $L=L_{-}$ around a black-hole attractor, and for counterrotating particles with momentum $L=-L_{+}$in black-hole and naked singularity geometries, respectively, and

$r_{b}^{(N S)-} \equiv 2 M+a-2 \sqrt{M} \sqrt{M+a}$ for $\mathbf{N S}: L=L_{-}$

for particles with $L=-L_{-}$orbiting in a naked singularity geometry. We have $\left\{r_{b}^{-}, r_{b}^{(N S)-}\right\} \in \Sigma_{\varepsilon}^{+}$.

The radii $\hat{r}_{ \pm} \in \Sigma_{\varepsilon}^{+}$, introduced in Sect. 3.2, for the circular orbits with zero angular momentum, are solutions of the equation $V^{\prime}=0$ with $L=0$ :

$$
\begin{aligned}
\hat{r}_{ \pm} & \equiv \frac{1}{\sqrt{6}}\left[\mathfrak{S} \pm \sqrt{\left.\frac{6 \sqrt{6} a^{2} M}{\mathfrak{S}}-\mathfrak{S}^{2}-6 a^{2}\right]}\right. \\
\mathfrak{S} & \equiv \sqrt{\frac{4 a^{4}}{\mathfrak{s}^{1 / 3}}+\mathfrak{s}^{1 / 3}-2 a^{2}}, \\
\mathfrak{s} & \equiv\left(27 M^{2} a^{4}-8 a^{6}+3 M \sqrt{81 M^{2} a^{8}-48 a^{10}}\right)
\end{aligned}
$$

in the naked singularity geometries of NSI-class (see also [19]). In the case of NSIa spacetimes, there are also orbits

\footnotetext{
$\overline{5}$ The spin $a=1.28112 M$ in Eq. (A.3) is introduced only for convenience in the definition of the functions $r_{l s c o}^{(\mathrm{NS})-}$, which can be matched for continuity in $a=1.28112 M$.
}

$r_{v}^{ \pm} \in \Sigma_{\varepsilon}^{+}$of counterrotating particles with $L=-L_{-}$and zero energy $\mathscr{E}=0$ :

$$
\begin{aligned}
r_{v}^{+} & \equiv \frac{4}{3} M\left(1+\sin \left[\frac{1}{3} \arcsin \left(1-\frac{27 a^{2}}{16 M^{2}}\right)\right]\right), \\
r_{v}^{-} & \equiv \frac{8}{3} M \sin \left[\frac{1}{6} \arccos \left(1-\frac{27 a^{2}}{16 M^{2}}\right)\right]^{2} .
\end{aligned}
$$

\section{Appendix B: Spacetime classes and limiting spins}

Interacting with the surrounding matter and fields, an attractor can pass, during its evolution, through stages of adjustment of its spin-spin-down or spin-up processes; see also $[5,7,10,44,56-61]$. These phenomena will involve also the interaction of the accretor with matter and fields in the ergoregion (see, for example, [1]). Questioning the possible disruption or formation of a horizon and the consequent formation and existence of a NS spacetime, it is obviously important to consider the possibility of a transition, through the BHIII and NSIa classes that could lead to a disruption of the event horizon. In this case, the matter dynamics in the BHIII and NSIa geometries with spins $a \approx M$ is especially relevant. For $a=M$ the effective potential is an increasing function of the radius orbits with $L \leq 0[6,51]$. There are circular orbits with $\left.L=L_{-} \in\right] 2 / \sqrt{3}, 1.68707[\mu M$. Figure 7 shows the analogies and differences between the orbits in the $\mathbf{B H}$ geometry with $a / M=1-\varepsilon$ and in the NS-geometry with $a / M=1+\varepsilon$ and $\varepsilon=10^{-5}$. The radius $\hat{r}_{+}$for naked singularities can be defined at any value $a=M+\varepsilon$ with $\varepsilon>0$; the black-hole counterpart $\hat{r}_{+}=M+f(\varepsilon)$, where $f(\varepsilon)<0$, can easily be evaluated and is of the order of $(a-M)^{2}$. The orbital structures in the two cases $a \lesssim M$ and $a \gtrsim M$ are completely different. Moreover, the radius $r_{v}^{+}$has a maximum at $a=M$. In Fig. 7, we show the angular momenta and energies of the orbits in two different regions. Figure 8 shows the effects of a transition of the black-hole geometries among the classes defined in Table 1 for a shift of the source spin in $a_{i} \in \mathscr{A}_{B H}$ of $\pm \varepsilon= \pm 0.01 \mathrm{M}$. The more significant change is in the structure of $\Sigma_{\varepsilon}^{+}$, a consequence of a shift $\pm \varepsilon_{a}$ of the spins $a_{b}^{-}=0.828427 M$ and $a_{2}=0.942809 M$. An analogous plot can be made for the case of a naked singularity; however, as can easily be seen in Fig. 4, the most relevant transitions between the sections of the dynamic structure of $\Sigma_{\varepsilon}^{+}$occur between NSIa and NSIb classes for a change in the geometry at $a=a_{\mu}$, with the resulting shift of the region $\Psi\left(-L_{-}\right)$into $\Sigma\left(L_{-}\right)$(for an increase of the attractor spin), between the classes NSIb and NSII for a change in the geometry at $a=a_{4}$, and the transition from $\Sigma\left(-L_{-}\right)$to $\Sigma\left(L_{-}\right)$ and from $\Sigma_{u}^{<}\left(L_{-}\right)$to $\Sigma_{s}\left(L_{-}\right)$. A thorough study of these sources will be the subject of further work. 

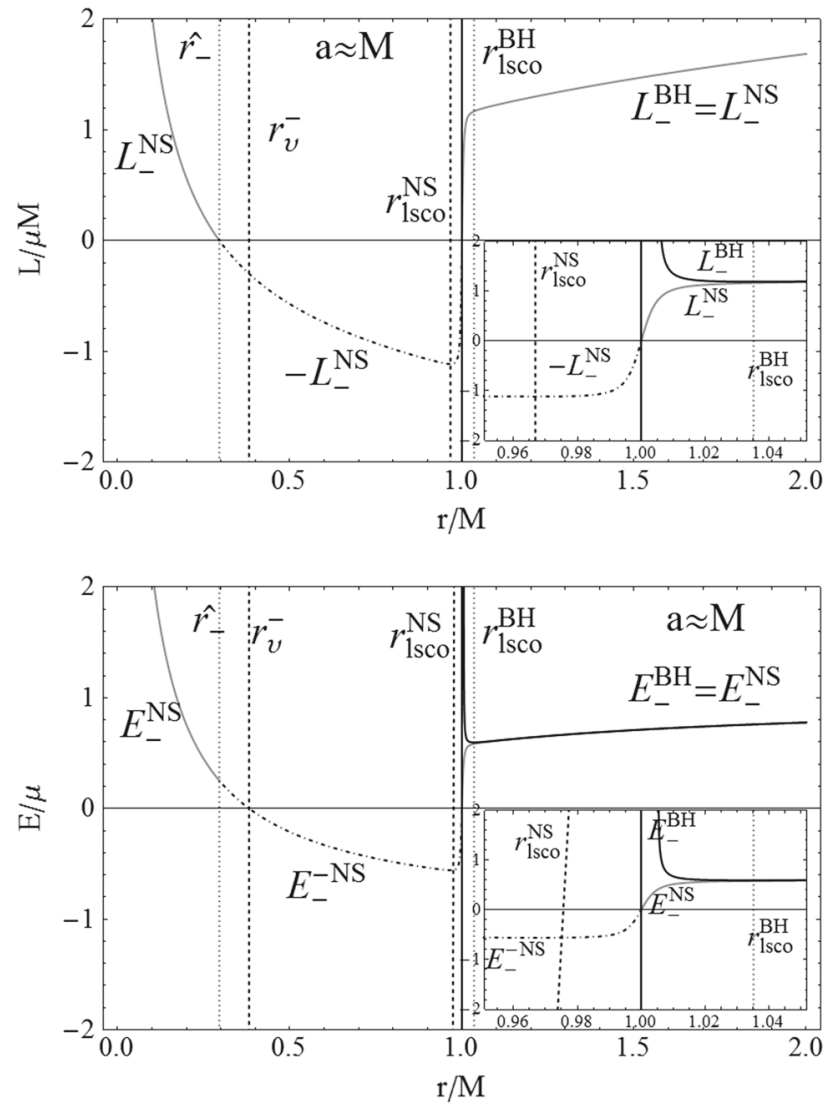

Fig. 7 Orbital energies and angular momenta in the $\mathbf{B H}$-case, with $a / M=1-\varepsilon$, and the NS-case, with $a / M=1+\varepsilon$ and $\varepsilon=10^{-5}$. To simplify the plots, we adopted a notation distinguishing explicitly the NS and BH cases: $E_{-}^{-N S} \equiv E\left(-L_{-}\right)$and $E_{-}^{N S} \equiv E\left(L_{-}\right)$for the naked singularity, $E_{-}^{B H}$ and $L_{-}^{B H}$ for the black hole. The marginally stable orbits are denoted $\left(r_{l s c o}^{B H}, r_{l s c o}^{N S}\right)$. The orbit $\hat{r}_{-}$, where $L=0$, is shown with a dashed line. We have $r_{l s c o}^{B H}=1.03523 M, r_{l s c o}^{N S}=0.966815 M$, and $\hat{r}_{+}=M, \hat{r}_{-}=0.295605 M$; inside the plots are zooms for the region close to the $\mathbf{B H}$ horizon

\section{Appendix C: The static limit}

We consider here in more detail the role of the static limit in the determination of the dynamical structure of the ergoregion, especially in the NS sector. In particular, we will analyze the behavior of the circular motion in the regions very close to $r_{\varepsilon}^{+}$, varying the spin of the attractor to determine its influence on the determination of the phases of equilibrium of the orbiting configurations. Particular attention is given to the analysis of the energy and momentum of the particle in orbit in those regions, as they are relevant for the orbital decay. We will show that both conserved quantities do not have a monotonous trend with spin, as the source evolves, implying the decay of the orbiting matter. This may favor those geometries where the energy or the orbital momentum is at a minimum.

The effective potential function $V(r ; L, a)$ given in Eq. (7) is well defined and positive at $r=r_{\varepsilon}^{+}$for $a>0$, it has

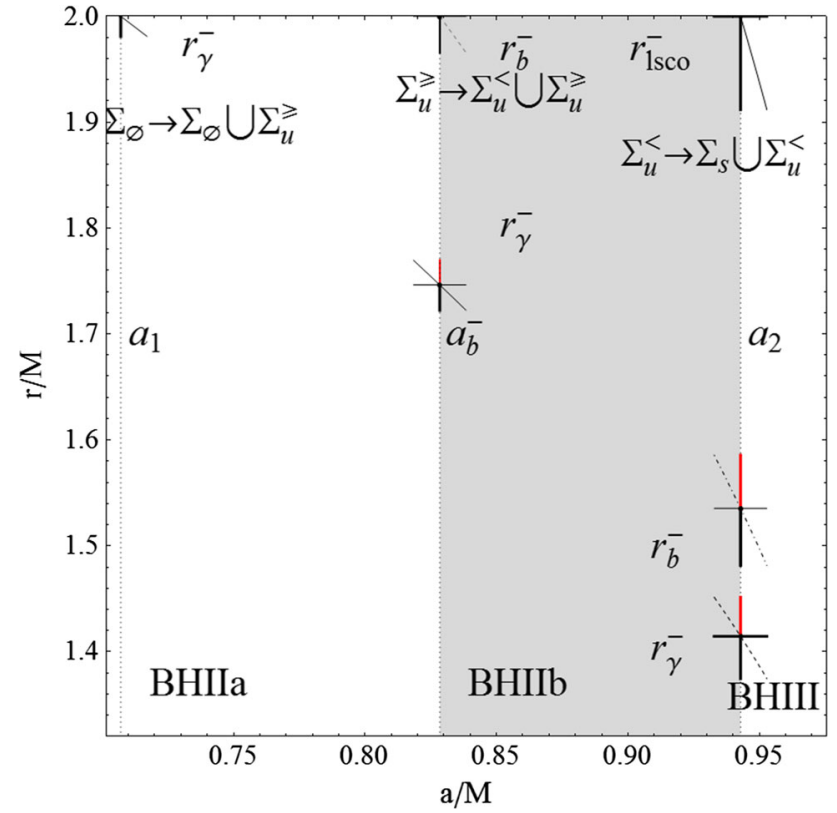

Fig. 8 Black-hole geometries in the region $\Sigma_{\varepsilon}^{+}$. The $\mathbf{B H}$ spin $a$ and the radius $r$ are in units of mass $M$. The BHIIa, BHIIb and BHIII classes are explicitly shown. Dotted lines represent the spins $a_{i} \in\left\{a_{1}, a_{b}^{-}, a_{3}\right\}$. The points correspond to the couples $\left(r_{\gamma}^{-}\left(a_{1}\right), a_{1}\right)$, where $r_{\gamma}^{-}\left(a_{1}\right)=r_{\varepsilon}^{+}$, $\left(r_{\gamma}^{-}\left(a_{b}^{-}\right), a_{b}^{-}\right)$and $\left(r_{b}^{-}\left(a_{b}^{-}\right), a_{b}^{-}\right)$, where $r_{b}^{-}\left(a_{b}^{-}\right)=r_{\varepsilon}^{+},\left(r_{\gamma}^{-}\left(a_{2}\right), a_{2}\right)$, $\left(r_{b}^{-}\left(a_{2}\right), a_{2}\right)$, and $\left(r_{l s c o}^{-}\left(a_{2}\right), a_{2}\right)$, where $r_{l s c o}^{-}\left(a_{2}\right)=r_{\varepsilon}^{+}$. The horizontal black lines crossing the points are $a_{i} \pm \varepsilon_{a}$, where $\varepsilon_{a}=1 / 100 M$. The vertical black lines are $r_{i}\left(a_{j}+\varepsilon_{a}\right)$ and the red vertical lines are $r_{i}\left(a_{j}-\varepsilon_{a}\right)$. The curves $r_{i} \in\left\{r_{\gamma}^{-}, r_{b}^{-}, r_{l s c o}^{-}\right\}$in the ranges $a_{i} \pm \varepsilon_{a}$ are plotted. Close to the limiting couples $\left(r_{\gamma}^{-}\left(a_{1}\right), a_{1}\right),\left(r_{b}^{-}\left(a_{b}^{-}\right), a_{b}^{-}\right)$, and $\left(r_{l s c o}^{-}\left(a_{2}\right), a_{2}\right)$, we show explicitly the transitions between different sections of $\Sigma_{\varepsilon}^{+}$. The relevant transitions are for a change at $a_{b}^{-}=0.828427 M$ and $a_{2}=0.942809 M$

an extreme point in the static limit, according to Eq. (9), for all Kerr geometries, except for BHI spacetimes. Therefore, at $r=r_{\varepsilon}^{+}$there is a circular orbit with angular momentum $L_{\varepsilon}^{+} \equiv L_{-}\left(r_{\varepsilon}^{+}\right)>0$ and energy $E_{\varepsilon}^{+} \equiv V\left(r_{\varepsilon}^{+}, L_{\varepsilon}^{+}\right)>0$, but in the spacetime with $a=a_{1}$ we have $r_{\gamma}^{-}=r_{\varepsilon}^{+}$, that is, the static limit coincides with the photon orbit. The timelike particle orbit $r_{\varepsilon}^{+}$is stable, i.e. $r_{\varepsilon}^{+} \in \Sigma_{s}$, in the BHIII, NSI, and NSII geometries. Then the static limit is a marginally stable circular orbit in spacetimes with spins $a=a_{2}$ and $a=a_{4}$, while $r_{\varepsilon}^{+} \in \Sigma_{\bar{u}}^{\geq}$in BHIIa and NSIIIb spacetimes. Moreover, in the geometries in $a=a_{b}^{-}$and $a=a_{b}^{N S}$ we have $r_{b}^{-}=r_{\varepsilon}^{+}$ and $r_{b}^{(\mathrm{NS})}=r_{\varepsilon}^{+}$, respectively. On these orbits, the particle energy is $\mathscr{E}=E_{-}=\mu$, and the static limit coincides with the marginally bounded orbit. These special orbits should be interpreted as the limit of an orbit that approaches $r_{\varepsilon}^{+}$from $\Sigma_{\varepsilon}^{+}$or the outer region. An observer at infinity will verify that a particle moving along an unstable orbit will eventually cross the static limit and fall into the source or escape, depending on the attractor. The static limit in the equatorial plane is independent of the spacetime spin and there- 

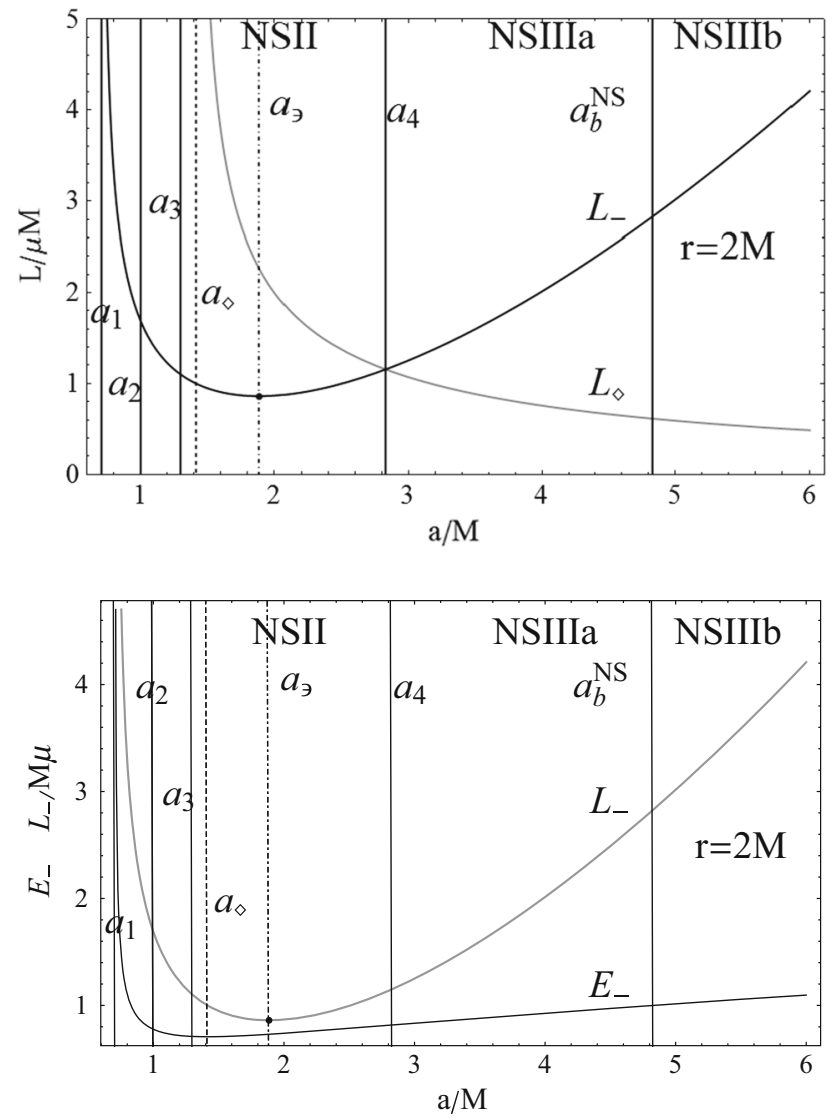

Fig. 9 Angular momentum and energy in the static limit $r_{\varepsilon}^{+}=2 M$. Bottom panel the orbital angular momentum $L_{-}$(gray curve) and the energy $E_{-}$(black curve) as functions of $a / M$. The minima are marked with points. The different $\mathbf{B H}$ and $\mathbf{N S}$ regions are also marked. We have $L_{-}>E_{-}$. Upper panel plot of $L_{\diamond}$ (gray curve) and $L_{-}$(black curve) versus the spin $a / M$. The asymptote at $a_{\diamond} \equiv \sqrt{2} M$ is plotted with a dashed line. The spin $a_{\ni} \equiv(4 \sqrt{2}) / 3 M \approx 1.88562 M$ is also plotted. At $L=L_{\diamond}$ the effective potential $V_{\varepsilon}^{+}$is constant with respect to a change of the attractor spin

fore it can be considered as invariant with respect to a slow change of the source spin (at constant $M$ ), but the constants of motion $\mathscr{E}$ and $\mathscr{L}$, instead, depend on the spin-mass ratio of the attractor and do not have a monotonically increasing behavior in the static limit with respect to a variation of the source spin; instead, they reach a minimum, as a function of $a / M$, in the NSII class of geometries. The energy function in the static limit decreases with the source spin for $a>a_{\diamond}$ and the particle angular momentum $L>L_{\diamond}$, where the rotation parameter is $a_{\diamond} \equiv \sqrt{2} M \approx 1.41421 M \in \mathbf{N S I I}$ and the angular momentum

$\frac{L_{\diamond}}{\mu M} \equiv 2 \sqrt{2} \sqrt{\frac{M^{2}}{a^{2}-2 M^{2}}}$

increases as the spacetime rotation decreases, and it diverges at $a=a_{\diamond}$, as shown in Fig. 9. When $L=L_{\diamond}$, the potential $V_{\varepsilon}^{+}$is constant, independently of the source spin. For lower spins, $a<a_{\diamond}$, regardless of the orbital angular momentum, the energy function increases with the spin of the $\mathbf{B H}$ or NS-sources. In the spacetime with $a=a_{\diamond}$, the particle energy in the static limit reaches a minimum value and, therefore, the particle energy increases as the spacetime rotation increases, until it reaches its asymptotic value for $a \rightarrow a_{1}$ (upper extreme of BHI sources), where we have indeed $r_{\varepsilon}^{+}=r_{\gamma}^{-}$(see Fig. 9). The particle orbital angular momentum has a minimum, as a function of the spin-mass ratio, in $a_{\ni} \equiv 2 a_{2} \approx 1.88562 M \in$ NSII, where $a_{3}<a_{\diamond}<a_{\ni}<a_{4}$.

\section{References}

1. D.L. Meier, Black Hole Astrophysics the Engine Paradigm (Springer, Berlin, Heidelberg, 2012)

2. V.P. Frolov, A. Zelnikov, Introduction to Black Hole Physics (Oxford University Press, Oxford, 2011)

3. Stuart L. Shapiro, Saul A. Teukolsky, Phys. Rev. Lett. 66, 994 (1991)

4. T.A. Apostolatos, K.S. Thorne, Phys. Rev. D 46, 2435 (1992)

5. T. Jacobson, T.P. Sotiriou, Phys. Rev. Lett. 103, 141101 (2009)

6. T. Jacobson, T.P. Sotiriou, J. Phys. Conf. Ser. 222, 012041 (2010)

7. E. Barausse, V. Cardoso, G. Khanna, Phys. Rev. Lett. 105, 261102 (2010)

8. B. Giacomazzo, L. Rezzolla, N. Stergioulas, Phys. Rev. D 84, 024022 (2011)

9. S. Chandrasekhar, Principles of Stellar Dynamics (Dover Publications, New York, 1942)

10. Z. Stuchlik, S. Hledik, K. Truparova, Class. Q. Grav. 28, 155017 (2011)

11. M.A. Abramowicz, M. Calvani, L. Nobili, Nature 302, 597 (1983)

12. J.A. Font, F. Daigne, Mon. Not. R. Astron. Soc. 334, 383 (2002)

13. O. Korobkin, E. Abdikamalov, N. Stergioulas, E. Schnetter, B. Zink, S. Rosswog, C.D. Ott, Mon. Not. R. Astron. Soc. 431(1), 354 (2013)

14. Z. Li, C. Bambi, JCAP 1303, 031 (2013)

15. P.S. Joshi, D. Malafarina, R. Narayan, Class. Q. Grav. 31, 015002 (2014)

16. P.S. Joshi, D. Malafarina, Int. J. Mod. Phys. D 20, 2641 (2011)

17. M. Patil, P.S. Joshi, Phys. Rev. D 84, 10 (2011)

18. M. Patil, P. Joshi, Class. Q. Grav. 28, 23 (2011)

19. D. Pugliese, H. Quevedo, R. Ruffini, Phys. Rev. D 84, 044030 (2011)

20. J. Gariel, N.O. Santos, J. Silk, Phys. Rev. D 90, 063505 (2014)

21. Z. Kovacs, T. Harko, Phys. Rev. D 82, 124047 (2010)

22. Z. Stuchlik, J. Schee, Class. Q. Grav. 30, 075012 (2013)

23. N. Pelavas, N. Neary, K. Lake, Class. Q. Grav. 18, 1319 (2001)

24. C. Herdeiro, E. Radu, Phys. Rev. D 89, 124018 (2014)

25. D. Pugliese, H. Quevedo, R. Ruffini, Phys. Rev. D 83, 024021 (2011)

26. D. Pugliese, H. Quevedo, R. Ruffini, Phys. Rev. D 83, 104052 (2011)

27. D. Pugliese, H. Quevedo, R. Ruffini, arXiv:1304.2940 [gr-qc]

28. D. Pugliese, H. Quevedo, R. Ruffini, Phys. Rev. D 88, 024042 (2013)

29. J. Schee, Z. Stuchlik, JCAP 1304, 005 (2013)

30. M. Patil, P.S. Joshi, Phys. Rev. D 82, 104049 (2010)

31. M. Stute, M. Camenzind, Mon. Not. R. Astron. Soc. 336, 831 (2002)

32. M.A. Abramowicz, P.C. Fragile, Liv. Rev. Relat. 16, 1 (2013) 
33. Q. Lei, M.A. Abramowicz, P.C. Fragile, J. Horak, M. Machida, O. Straub, Astron. Astrophys. 498, 471 (2009)

34. D. Pugliese, G. Montani, Phys. Rev. D 91(8), 083011 (2015)

35. S.W. Hawking, G.F.R. Ellis, The Large Scale Structure of SpaceTime (Cambridge University Press, Cambridge, 1973)

36. R. Penrose, R.M. Floyd, Science 229, 177-179 (1971)

37. R.S.S. Vieira, J. Schee, W. Kluźniak, Z. Stuchlík, M. Abramowicz, Phys. Rev. D 90, 024035 (2014)

38. P. Slaný, Z. Stuchlík, Class. Q. Grav. 22, 3623-3651 (2005)

39. H. Kučáková, P. Slaný, Z. Stuchlík, J. Cosmol. Astropart. Phys. 1, $033(2011)$

40. L.D. Landau, E.M. Lifshitz, The Classical Theory of Fields (Pergamon Press, Oxford, 1980)

41. J.B. Griffiths, J. Podolský, Exact Space-Times in Einstein's General Relativity (Cambridge University Press, Cambridge, 2009)

42. C.W. Misner, K.S. Thorne, J.A. Wheeler, Gravitation (Freeman, San Francisco, 1973)

43. R. Ruffini, in Black Holes $\stackrel{\circ}{U}$ Les Astres Occlus, ed. by C. DeWitt, B.S. DeWitt (Gordon and Breach, New York, 1973)

44. R.M. Wald, Ann. Phys. 82, 548 (1974)

45. R.M. Wald, General Relativity (The University of Chicago Press, Chicago, 1984)

46. K. Adámek, Z. Stuchlík, Class. Q. Grav. 30, 205007 (2013)

47. D. Pugliese, G. Montani, M.G. Bernardini, Mon. Not. R. Astron. Soc. 428(2), 952 (2013)

48. R.H. Boyer, Proc. Camb. Philos. Soc. 61, 527 (1965)

49. V. Cardoso, P. Pani, M. Cadoni, M. Cavaglia, Phys. Rev. D 77(124), 044 (2008)

50. N. Comins, B.F. Schutz, Proc. R. Soc. A 364(1717), 211-226 (1978)

51. Z. Li, C. Bambi, Phys. Rev. D 87, 124022 (2013)

52. Z. Stuchlik, Bull. Astron. Inst. Czech. 31, 129 (1980)

53. J. Biéak, Z. Stuchlík, V. Balek, Bull. Astron. Inst. Czech. 40(2), 65-92 (1989)

54. V. Balek, J. Bicak, Z. Stuchlik, Bull. Astron. Inst. Czech. 40(3), 133-165 (1989)

55. J. Kovar, O. Kopacek, V. Karas, Z. Stuchlik, Class. Q. Grav. 27, 135006(2010)
56. S. Gao, Y. Zhang, Phys. Rev. D 87(4), 044028 (2013)

57. Z. Stuchlik, Bull. Astron. Inst. Czech. 32, 68 (1981)

58. M.H.P.M. van Putten, Prog. Theor. Phys. 127(2), 331-354 (2012)

59. C.F. Gammie, S.L. Shapiro, J.C. McKinney, Astrophys. J. 602, 312 (2004)

60. P. Abolmasov, Mon. Not. R. Astron. Soc. 432, 761-768 (2013)

61. M. Kesden, Phys. Rev. D 83, 104011 (2011)

62. O. Luongo, H. Quevedo, Phys. Rev. D 90, 084032 (2014)

63. R.M. Wald, V. Iyer, Phys. Rev. D 44, 3719 (1991)

64. P.S. Joshi, Gravitational Collapse and Spacetime Singularities (Cambridge Monographs on Mathematical Physics, New York, 2007)

65. P. Pradhan, P. Majumdar, Eur. Phys. J. C 73(6), 2470 (2013)

66. R. Znajek, Nature 262, 270 (1976)

67. A.R. Prasanna, R.K. Varma, Pramana 8(3), 229 (1977)

68. A.R. Prasanna, C.V. Vishveshwara, Pramana 11, 359 (1978)

69. A.N. Aliev, N. Özdemir, Mon. Not. R. Astron. Soc. 336, 241 (2002)

70. G. Preti, Class. Q. Grav. 21, 3433 (2004)

71. P. Bakala, E. Šrámková, Z. Stuchlík, G. Török, Class. Q. Grav. 27, 045001 (2010)

72. V.P. Frolov, A.A. Shoom, Phys. Rev. D 82, 084034 (2010)

73. T. Igata, T. Harada, M. Kimura, Phys. Rev. D 85, 104028 (2012)

74. R. Shiose, M. Kimura, T. Chiba, Phys. Rev. D 90, 124016 (2014)

75. S. Hussain, I. Hussain, M. Jamil, Eur. Phys. J. C 74(12), 3210 (2014)

76. R.D. Blandford, R.L. Znajek, Mon. Not. R. Astron. Soc. 179, 433 (1977)

77. K.S. Thorne, R.H. Price, D.A. Macdonald, Black Holes: The Membrane Paradigm (Yale University, New Haven, 1986)

78. A. Sadowski, R. Narayan, A. Tchekhovskoy, D. Abarca, Y. Zhu, J.C. McKinney, Mon. Not. R. Astron. Soc. 447, 49 (2015)

79. L. Zoltan Kelley, A. Tchekhovskoy, R. Narayan, Mon. Not. R. Astron. Soc. 445, 3519 (2014)

80. S.M. Wagh, N. Dadhich, Phys. Rep. 183(4), 137-192 (1989)

81. N. Dadhich, arXiv:1210.1041 